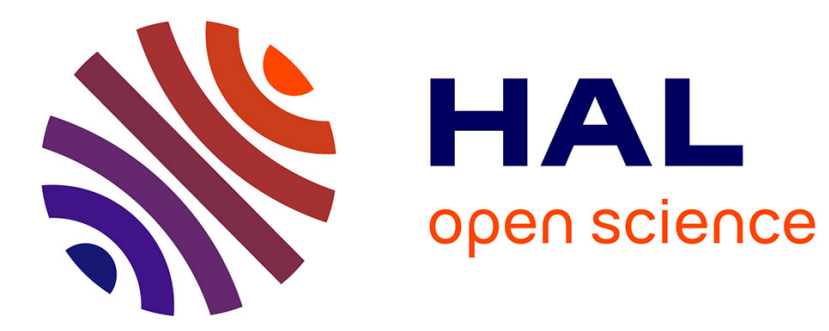

\title{
Circular edge singularities for the Laplace equation and the elasticity system in 3-D domains
}

\author{
Zohar Yosibash, Samuel Shannon, Monique Dauge, Martin Costabel
}

\section{To cite this version:}

Zohar Yosibash, Samuel Shannon, Monique Dauge, Martin Costabel. Circular edge singularities for the Laplace equation and the elasticity system in 3-D domains. International Journal of Fracture, 2011, 168, pp.31-52. 10.1007/s10704-010-9553-y . hal-00490971v2

\section{HAL Id: hal-00490971 \\ https://hal.science/hal-00490971v2}

Submitted on 8 Oct 2010

HAL is a multi-disciplinary open access archive for the deposit and dissemination of scientific research documents, whether they are published or not. The documents may come from teaching and research institutions in France or abroad, or from public or private research centers.
L'archive ouverte pluridisciplinaire HAL, est destinée au dépôt et à la diffusion de documents scientifiques de niveau recherche, publiés ou non, émanant des établissements d'enseignement et de recherche français ou étrangers, des laboratoires publics ou privés. 


\title{
CIRCULAR EDGE SINGULARITIES FOR THE LAPLACE EQUATION AND THE ELASTICITY SYSTEM IN 3-D DOMAINS
}

\author{
ZOHAR YOSIBASH, SAMUEL SHANNON, MONIQUE DAUGE AND MARTIN COSTABEL
}

\begin{abstract}
Asymptotics of solutions to the Laplace equation with Neumann or Dirichlet conditions in the vicinity of a circular singular edge in a three-dimensional domain are derived and provided in an explicit form. These asymptotic solutions are represented by a family of eigen-functions with their shadows, and the associated edge flux intensity functions (EFIFs), which are functions along the circular edge. We provide explicit formulas for a penny-shaped crack for an axisymmetric case as well as a case in which the loading is non-axisymmetric. Explicit formulas for other singular circular edges such as a circumferential crack, an external crack and a $3 \pi / 2$ reentrant corner are also derived.

The mathematical machinery developed in the framework of the Laplace operator is extended to derive the asymptotic solution (three-component displacement vector) for the elasticity system in the vicinity of a circular edge in a three-dimensional domain. As a particular case we present explicitly the series expansion for a traction free or clamped penny-shaped crack in an axisymmetric or a non-axisymmetric situation.

The precise representation of the asymptotic series is required for constructing benchmark problems with analytical solutions against which numerical methods can be assessed, and to develop new extraction techniques for the edge flux/intensity functions which are of practical engineering importance in predicting crack propagation.
\end{abstract}

\section{CONTENTS}

1. Introduction.

2. Asymptotic solution for the Laplace equation

2.1. Axi-symmetric case

2.2. General case

3. Asymptotic solution for the elasticity system

3.1. Homogeneous boundary conditions 18

3.2. Axi-symmetric case 19

3.3. Non axi-symmetric case 23

4. Summary and Conclusions 26

Appendix A. Derivation of the shadow terms associated with $\alpha=0$ for the traction free, $\begin{array}{ll}\text { axi-symmetric penny-shaped crack } & 27\end{array}$

$\begin{array}{ll}\text { References } & 28\end{array}$

$\begin{array}{lr}\text { Addresses } & 28\end{array}$

\section{INTRODUCTION.}

Solutions of elliptic boundary value problems over two dimensional domains, for example those arising in heat transfer and elasticity, when posed and solved in non-smooth domains like polygons, have non-smooth parts. These are described in terms of special singular functions depending on the geometry and the differential operators on one hand, and of unknown coefficients depending on the given right hand side and boundary conditions on the other hand, see e.g. [12]. 
In three dimensional domains as polyhedra both vertex and edge singularities exist, see [4, 9]. For straight edges we have provided explicit representation of the singular solutions $[3,8,13]$ as a series characterized by:

- an exponent $\alpha$ which belongs to a discrete set $\left\{\alpha_{k}, k \in \mathbb{N}\right\}$ of eigen-values depending only on the geometry and the operator, and which determines the level of non-smoothness of the singularity. Any eigen-value $\alpha_{k}$ is computed by solving a 2-D problem.

- eigen-functions $\phi_{k, 0}(\varphi)$ which depends on the geometry of the domain and the operator. These eigen-functions are computed by solving a set of 2-D problems.

- a function along the edge, denoted by $A_{k}(s)$ ( $s$ is a coordinate along the edge) and called "Edge Flux/Stress Intensity Function" (EFIF/ESIF) which determines the "amount of energy" residing in each singularity.

Here we concentrate on circular edges (a "penny-shaped crack" being a special renown case) in 3D domain, and derive explicitly singular series expansion in the vicinity of such an edge first for the simplest scalar elliptic operator, the Laplace operator, and then for the elasticity system. We demonstrate that our asymptotic solution for the elasticity system in the simplified case of the penny-shaped crack under axi-symmetric boundary conditions and geometry reduces to the one presented in [7].

From the engineering perspective the edge flux/stress intensity functions $A_{k}(s)$ (EFIFs/ESIFs) when $\alpha_{k}<1$ ( $\alpha_{1}=\frac{1}{2}$ for the penny-shaped crack) are of major importance. These are used to predict failure initiation and propagation, and are an important ingredient in any failure law for cracked and V-notched structures. To efficiently and accurately compute them, the asymptotic solution has first to be explicitly derived.

This work is motivated by the need to compute edge stress intensity functions (ESIFs) for elasticity problems in 3-D domains. These are of significant engineering importance in cracked and V-notched structures, in which the ESIFs may (and often do) vary along the crack front.

In order to explain the ideas of the implementation of the method and to test its efficiency, we consider the Laplace operator first. This is a simpler elliptic operator that allows more transparent analytic computations and invokes all necessary characteristics of the elasticity system. Thus, the characteristics of the solution can be more easily addressed.

The first three singular terms for the solution of the Laplace equation in the vicinity of a circular edge with homogeneous Dirichlet boundary conditions were analyzed from a theoretical viewpoint in [10]. The first two terms in the Neumann case are provided in [1] when the edge is the boundary of a smooth plane crack surface. For the elasticity system, Leblond\&Torlai [6] provided the machinery for the pointwise derivation of the solution up to second order for a general curved crack, whereas Leung\&Su derived the asymptotic series for the axi-symmetric case in [7]. Herein, we present a different approach enabling the computation of the entire series solution up to an arbitrary order for any circular edge be it in an axi-symmetric or non-axisymmetric setting. This explicit representation illustrates the distinct two levels of complexity of shadow terms associated with the curved singular edge.

The Laplace equation and the notation are introduced in section 2. The systematic derivation of the singular series expansion is presented for homogeneous Dirichlet and Neumann boundary conditions. Both axi-symmetric and non-axi-symmetric configurations are addressed, and for a penny-shaped crack, a circumferential crack, an external crack and a $3 \pi / 2$ reentrant corner we also present closed form explicit singular series expansion. The asymptotic expansion is provided in terms of eigen-functions, their shadows, the EFIFs and their derivatives.

We then use the machinery developed in the framework of the Laplace equation to provide explicit representation of similar cases associated with the elasticity system (with clamped or traction free boundary conditions) in section 3. The elasticity explicit asymptotic solution is mandatory for the computation of edge stress intensity functions for cracks occurring usually in pipes and pressure vessels. 


\section{ASYMPTOTIC SOLUTION FOR THE LAPLACE EQUATION}

As a model, we choose a domain generated by rotating the 2-D plane $\Omega$ having a reentrant corner with an opening $\omega \in(0,2 \pi]$ (the case of a crack, $\omega=2 \pi$, is included) along the axis $x_{3}$, as shown in Figure 1 . The cylindrical coordinate system $r, \theta, x_{3}$ and the coordinate system attached to the circular edge $\rho, \varphi, \theta$ are shown in Figure 1. It is important to emphasize that domain's geometry does not need

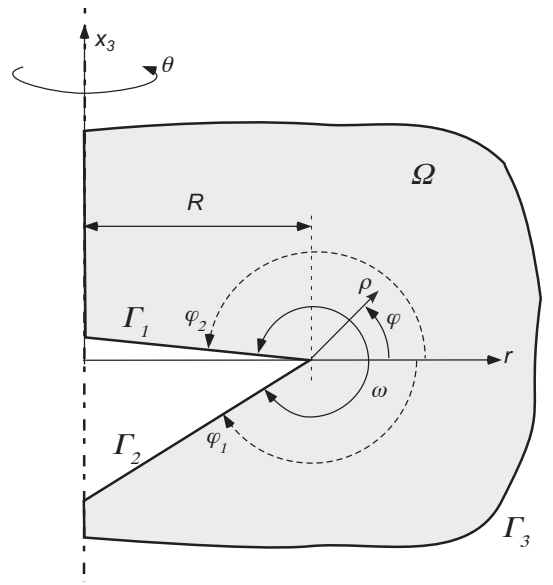

FIGURE 1. Model domain of interest $\Omega$ and the coordinate systems.

to be axi-symmetric, but only the generated circular singular edge. An example of several different circular singular edges to which the analysis in this manuscript is applicable are shown in Figure 2. For example, the lower singular edge in Figure 2 (a) is determined by $\varphi \in(-\pi, \pi / 2)$, the outer circular crack in Figure 2 (b) is determined by $\varphi \in(0,2 \pi)$ whereas the penny-shaped crack in (c) is determined by $\varphi \in(-\pi, \pi)$. Finally the re-entrant corner with the solid angle $\omega$ in Figure 2 (d) is determined by $\varphi \in((\pi-\omega) / 2,(\pi+\omega) / 2)$.
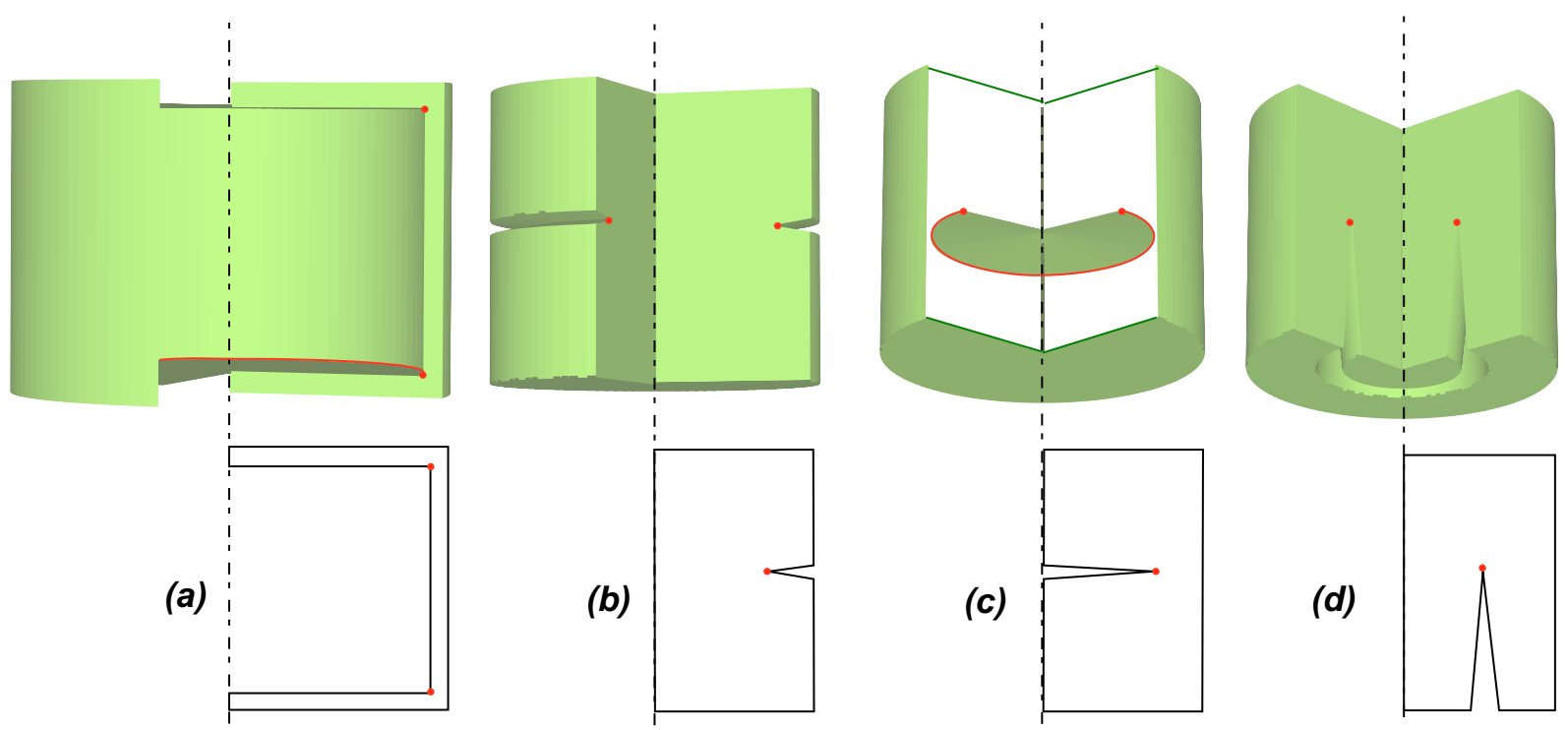

FIGURE 2. Different types of singular circular edges (only a sector is plotted so the circular edge is clearly visible. The domain in (c) includes the renown "penny-shaped" crack. 
For the Laplace operator, we are interested in solutions $\tau(\boldsymbol{x})$ of the equation:

$$
\triangle^{3 D} \tau \stackrel{\text { def }}{=}\left(\partial_{r r}+\frac{1}{r} \partial_{r}+\frac{1}{r^{2}} \partial_{\theta \theta}+\partial_{33}\right) \tau=0,
$$

where $\partial_{r} \stackrel{\text { def }}{=} \frac{\partial}{\partial r}, \partial_{r r} \stackrel{\text { def }}{=} \frac{\partial^{2}}{\partial r^{2}} \partial_{\theta \theta} \stackrel{\text { def }}{=} \frac{\partial^{2}}{\partial \theta^{2}}$ and $\partial_{33} \stackrel{\text { def }}{=} \frac{\partial^{2}}{\partial x_{3}^{2}}$. Homogeneous Dirichlet or Neumann boundary conditions are considered on $\Gamma_{1} \times[0,2 \pi]$ and $\Gamma_{2} \times[0,2 \pi]$.

The solution in the vicinity of the edge is of interest so we perform a change of coordinates as follows:

$$
r=\rho \cos \varphi+R, \quad x_{3}=\rho \sin \varphi .
$$

The Laplace operator in the new coordinates is given by:

$$
\triangle^{3 D}=\partial_{\rho \rho}+\frac{1}{\rho} \partial_{\rho}+\frac{1}{\rho^{2}} \partial_{\varphi \varphi}+\frac{1}{r}\left[\cos \varphi \partial_{\rho}-\frac{1}{\rho} \sin \varphi \partial_{\varphi}\right]+\frac{1}{r^{2}} \partial_{\theta \theta}
$$

2.1. Axi-symmetric case. For an axi-symmetric domain and boundary conditions the solution is independent of $\theta$. Then the last term in (3) vanishes and the Laplace operator for $\rho / R \ll 1$ reads:

$$
\triangle^{A x i}=\partial_{\rho \rho}+\frac{1}{\rho} \partial_{\rho}+\frac{1}{\rho^{2}} \partial_{\varphi \varphi}+\frac{1}{r}\left[\cos \varphi \partial_{\rho}-\frac{1}{\rho} \sin \varphi \partial_{\varphi}\right] .
$$

Remark 1. Since $r \rightarrow \infty$ as $R \rightarrow \infty$, one may observe that $\triangle^{A x i} \stackrel{R \rightarrow \infty}{\longrightarrow} \triangle^{2 D}$.

Axisymmetric solutions $\tau$ of (1) are equivalently the solutions of $\frac{r}{R} \triangle^{A x i} \tau=0$, i.e.

$$
\left(1+\frac{\rho}{R} \cos \varphi\right)\left[\partial_{\rho \rho}+\frac{1}{\rho} \partial_{\rho}+\frac{1}{\rho^{2}} \partial_{\varphi \varphi}\right] \tau+\frac{1}{R}\left[\cos \varphi \partial_{\rho}-\frac{1}{\rho} \sin \varphi \partial_{\varphi}\right] \tau=0 .
$$

Multiplying by $\rho^{2}$, we find another equivalent equation

$$
\left[\left(\rho \partial_{\rho}\right)^{2}+\partial_{\varphi \varphi}\right] \tau+\frac{\rho}{R}\left[\cos \varphi\left(\rho \partial_{\rho}\right)-\sin \varphi \partial_{\varphi}+\cos \varphi\left(\left(\rho \partial_{\rho}\right)^{2}+\partial_{\varphi \varphi}\right)\right] \tau=0 .
$$

The solution in the vicinity of the singular point in the 2-D cross-section $\Omega$ can be obtained in a simple form as an asymptotic series defined by eigen-pairs of a one-dimensional boundary value problem on the interval $\varphi \in\left(\varphi_{1}, \varphi_{1}+\omega\right)$. If we denote one such eigen-pair by $\alpha$ and $\phi_{0}(\varphi)$, then it is conceivable (as to be shown in the sequel) that for the axi-symmetric case a solution is formed as an asymptotic series of the form:

$$
\tau=A \rho^{\alpha} \sum_{i=0}^{\infty}\left(\frac{\rho}{R}\right)^{i} \phi_{i}(\varphi)
$$

Boundary Conditions: To satisfy the homogeneous boundary conditions, the series representation has to satisfy the following constraints on $\varphi=\varphi_{1}$ and $\varphi=\varphi_{2}=\varphi_{1}+\omega$ :

$$
\begin{array}{ll}
\phi_{i}\left(\varphi=\varphi_{1}, \varphi_{2}\right)=0 & \text { in Dirichlet case } \\
\phi_{i}^{\prime}\left(\varphi=\varphi_{1}, \varphi_{2}\right)=0 & \text { in Neumann case }
\end{array}
$$

Substitute (7) in (6) to obtain:

$$
\begin{aligned}
A & \left\{\left[\alpha^{2} \phi_{0}+\phi_{0}^{\prime \prime}\right]\right. \\
& +\frac{\rho}{R}\left[\left((\alpha+1)^{2} \phi_{1}+\phi_{1}^{\prime \prime}\right)+\alpha \cos \varphi \phi_{0}-\sin \varphi \phi_{0}^{\prime}+\cos \varphi\left(\alpha^{2} \phi_{0}+\phi_{0}^{\prime \prime}\right)\right] \\
& +\frac{\rho^{2}}{R^{2}}\left[\left((\alpha+2)^{2} \phi_{2}+\phi_{2}^{\prime \prime}\right)+(\alpha+1) \cos \varphi \phi_{1}-\sin \varphi \phi_{1}^{\prime}+\cos \varphi\left((\alpha+1)^{2} \phi_{1}+\phi_{1}^{\prime \prime}\right)\right] \\
& +\frac{\rho^{3}}{R^{3}}\left[\left((\alpha+3)^{2} \phi_{3}+\phi_{3}^{\prime \prime}\right)+(\alpha+2) \cos \varphi \phi_{2}-\sin \varphi \phi_{2}^{\prime}+\cos \varphi\left((\alpha+2)^{2} \phi_{2}+\phi_{2}^{\prime \prime}\right)\right] \\
& +\cdots\}=0
\end{aligned}
$$


To satisfy the above equation for any $A$ and $\rho$, the following relationships must hold:

$$
\begin{aligned}
\alpha^{2} \phi_{0}+\phi_{0}^{\prime \prime} & =0 \\
(\alpha+1)^{2} \phi_{1}+\phi_{1}^{\prime \prime} & =-\left(\alpha \cos \varphi \phi_{0}-\sin \varphi \phi_{0}^{\prime}\right)-\cos \varphi\left(\alpha^{2} \phi_{0}+\phi_{0}^{\prime \prime}\right) \\
(\alpha+2)^{2} \phi_{2}+\phi_{2}^{\prime \prime} & =-\left((\alpha+1) \cos \varphi \phi_{1}-\sin \varphi \phi_{1}^{\prime}\right)-\cos \varphi\left((\alpha+1)^{2} \phi_{1}+\phi_{1}^{\prime \prime}\right) \\
(\alpha+3)^{2} \phi_{3}+\phi_{3}^{\prime \prime} & =-\left((\alpha+2) \cos \varphi \phi_{2}-\sin \varphi \phi_{2}^{\prime}\right)-\cos \varphi\left((\alpha+2)^{2} \phi_{2}+\phi_{2}^{\prime \prime}\right)
\end{aligned}
$$

Substituting the RHS of equation (11) in (12) one obtains:

$$
\begin{aligned}
\alpha^{2} \phi_{0}+\phi_{0}^{\prime \prime}= & 0, \quad \varphi_{1}<\varphi<\varphi_{2} \\
(\alpha+1)^{2} \phi_{1}+\phi_{1}^{\prime \prime}= & -\left(\alpha \cos \varphi \phi_{0}-\sin \varphi \phi_{0}^{\prime}\right), \quad \varphi_{1}<\varphi<\varphi_{2} \\
(\alpha+i)^{2} \phi_{i}+\phi_{i}^{\prime \prime}= & -\left[(\alpha+i)(\alpha+i-1) \cos \varphi \phi_{i-1}-\sin \varphi \phi_{i-1}^{\prime}+\cos \varphi \phi_{i-1}^{\prime \prime}\right] \\
& i \geq 2, \quad \varphi_{1}<\varphi<\varphi_{2}
\end{aligned}
$$

These equations have to be completed by the boundary conditions (8) or (9).

Note the following:

- The equation (15) with BCs (8) or (9) is the one dimensional eigenvalue problem corresponding to the 2-D problem over $\Omega$, with eigen-value $\alpha$ and eigen-function $\phi_{0}$. Traditionally $\phi_{0}$ is called primal eigen-function.

- A recursive system of ordinary differential equations is obtained - once $\phi_{0}$ is computed from (15) it can be inserted in (16) to obtain $\phi_{1}$ then these both can be inserted in (17) to obtain $\phi_{2}$, etc.

- Only particular solutions in (16) and (17) are required.

Because (7) corresponds only to one representative eigen-pair, the complete solution should be a sum over all eigen-pairs $\alpha_{k}, \phi_{k, i}$, thus is a double sum series:

$$
\tau=\sum_{k} A_{k} \rho^{\alpha_{k}} \sum_{i=0}^{\infty}\left(\frac{\rho}{R}\right)^{i} \phi_{k, i}(\varphi)
$$

Remark 2. For each eigen-function and shadow $\phi_{k, i}(\varphi)$ the first index $k$ represents the eigen-value $\alpha_{k}$ to which this eigen-function is associated, whereas the second index $i \geq 1$ represents the rank of the shadow terms. Here $\alpha_{k}=\frac{k \pi}{\omega}$, where $k=0,1,2, \ldots$ for homogeneous Neumann BCs, and $k=1,2,3, \ldots$ for homogeneous Dirichlet BCs.

Remark 3. If $\alpha+1$ is not an eigenvalue of equation (15) with BCs (8) or (9), there exists a unique solution $\Phi_{1}$ to equation (16). On the other hand, if $\alpha+1$ is itself an eigenvalue, then it can either happen that (16) has no solution (then the ansatz (7) has to be completed with logarithmic terms), or (16) has infinitely many solutions. The same situation holds for equation (17), depending on whether $\alpha+i$ is an eigenvalue or not.

In the special case of a crack, we have $\alpha_{k}=\frac{k}{2}$, therefore resonances (i.e. $\alpha+i$ is an eigenvalue) always occur. Nevertheless, as proved in [2], logarithmic terms never appear: Equations (16) and (17) with Dirichlet or Neumann BCs are always solvable. An orthogonality condition against the eigenvector makes the solution unique, see (20).

2.1.1. A specific example problem - penny-shaped crack with axisymmetric loading and homogeneous Neumann BCs. As an example problem, consider a penny-shaped crack (Figure 2(c)), $\varphi_{1}=-\pi$, $\omega=2 \pi\left(\varphi_{2}=\pi\right)$ in an axisymmetric domain. For the crack in a 2-D cross-section with homogeneous Neumann BCs the following 2-D eigen-pairs are known - they are obtained by solving (15) complemented by BCs (9): 


\begin{tabular}{c|c|c}
$k$ & $\alpha_{k}$ & $\phi_{k, 0}$ \\
\hline 0 & 0 & 1 \\
1 & $\frac{1}{2}$ & $\sin \frac{\varphi}{2}$ \\
2 & 1 & $\cos \varphi$ \\
3 & $\frac{3}{2}$ & $\sin \frac{3 \varphi}{2}$ \\
4 & 2 & $\cos 2 \varphi$
\end{tabular}

TABLE 1. First four eigen-pairs for a crack with homogeneous Neumann BCs.

Note that the angular part of the first singular function is $\sin \frac{\varphi}{2}$ instead of the "usual" formula $\cos \frac{\varphi}{2}$ : This is due to the choice of the angular coordinate $\varphi \in(-\pi, \pi)$ instead of $\varphi \in(0,2 \pi)$.

Equations (15)-(17) can be solved (cf. Remark 3) for $\alpha_{k}=0,1 / 2,1,3 / 2$, obtaining $\phi_{0, i}, \phi_{1, i}, \phi_{2, i}$, $\phi_{3, i}$. They yield the following series solution for a penny-shaped crack with homogeneous Neumann BCs:

$$
\begin{aligned}
\tau= & A_{0} \\
+ & A_{1} \rho^{\frac{1}{2}}\left[\sin \frac{\varphi}{2}+\left(\frac{\rho}{R}\right) \frac{1}{4} \sin \frac{\varphi}{2}+\left(\frac{\rho}{R}\right)^{2}\left(\frac{1}{12} \sin \frac{\varphi}{2}-\frac{3}{32} \sin \frac{3 \varphi}{2}\right)\right. \\
& \left.\quad+\left(\frac{\rho}{R}\right)^{3}\left(\frac{1}{16} \sin \frac{\varphi}{2}-\frac{1}{30} \sin \frac{3 \varphi}{2}+\frac{5}{128} \sin \frac{5 \varphi}{2}\right)+\cdots\right] \\
+ & A_{2} \rho\left[\cos \varphi-\left(\frac{\rho}{R}\right) \frac{1}{4}+\left(\frac{\rho}{R}\right)^{2} \frac{3}{16} \cos \varphi-\left(\frac{\rho}{R}\right)^{3}\left(\frac{9}{128}+\frac{5}{64} \cos 2 \varphi\right)+\cdots\right] \\
+ & A_{3} \rho^{\frac{3}{2}}\left[\sin \frac{3 \varphi}{2}-\left(\frac{\rho}{R}\right) \frac{1}{4} \sin \frac{\varphi}{2}-\left(\frac{\rho}{R}\right)^{2} \frac{1}{32}\left(3 \sin \frac{\varphi}{2}-\frac{16}{5} \sin \frac{3 \varphi}{2}\right)\right. \\
& \left.\quad+\left(\frac{\rho}{R}\right)^{3}\left(-\frac{3}{40} \sin \frac{\varphi}{2}+\frac{5}{128} \sin \frac{3 \varphi}{2}-\frac{3}{70} \sin \frac{5 \varphi}{2}\right)+\cdots\right] \\
+ & \ldots \quad
\end{aligned}
$$

It is worthwhile to notice that we enforced the following orthogonality conditions on the shadow terms

$$
\int_{\varphi_{1}=-\pi}^{\varphi_{2}=\pi} \phi_{k, i}(\varphi) \phi_{k+i, 0}(\varphi) d \varphi=0, \quad k=0,1,2,3 \text { and } i=1,2,3
$$

making them unique.

One may notice that for $R \rightarrow \infty$ (the crack edge curvature tends to zero) only the first terms are non-zero so the solution (19) reduces to the 2-D solution:

$$
\tau \stackrel{R \rightarrow \infty}{\longrightarrow} A_{0}+A_{1} \rho^{1 / 2} \sin \frac{\varphi}{2}+A_{2} \rho \cos \varphi+A_{3} \rho^{3 / 2} \sin \frac{3 \varphi}{2}+\cdots
$$

Remark 4. The eigen-functions and shadows associated with $A_{0}$ and $A_{2}$ above are polynomials in local Cartesian variables $z_{1}:=\rho \cos \varphi$ and $z_{2}=\rho \sin \varphi$. This can be predicted by the general theory [5, 4].

To verify the correctness of the solution (19) we consider a torus with an inner radius $r_{1}=1.5$ and an outer radius $r_{2}=2.5$ having a circular crack with the tip at $R=2$, see Figure 3 .

Taking $A_{1}=1$ and $A_{k}=0, k \neq 1$, (notice that the outer boundary of the torus is $\rho_{\text {out }}=1 / 2$ and $\rho / R=1 / 4$ in the considered example) we prescribed on the outer surface of the torus Dirichlet 

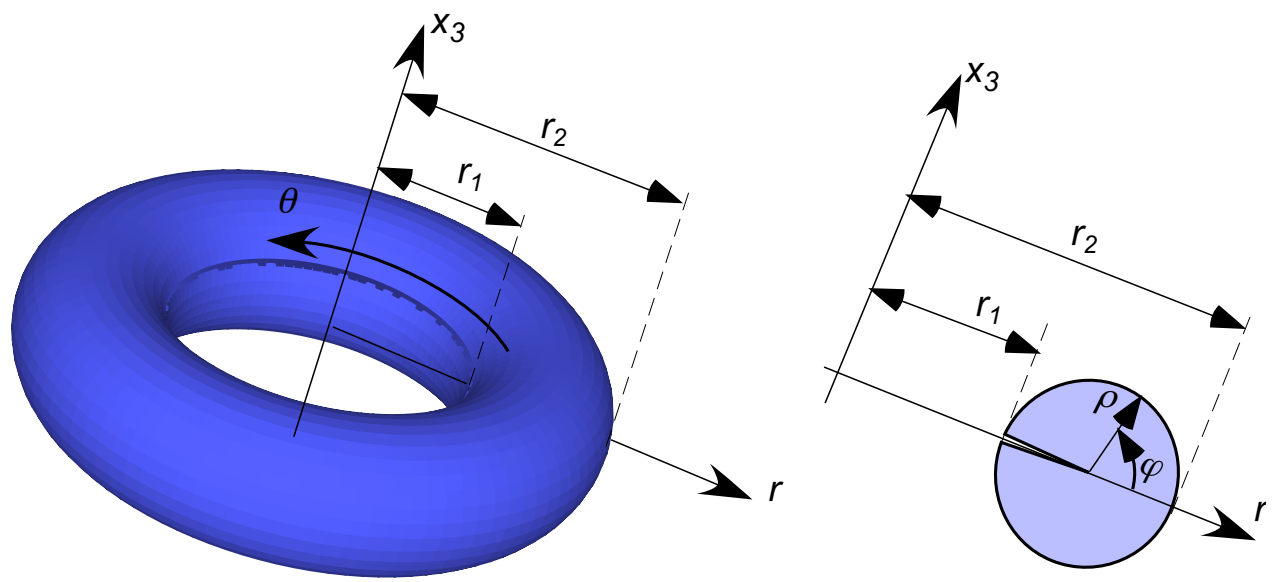

FIGURE 3. The axi-symmetric domain of interest (torus).

boundary conditions according to (19):

$$
\begin{aligned}
& \tau=\sqrt{\frac{1}{2}}\left[\sin \frac{\varphi}{2}+\left(\frac{1}{4}\right) \frac{1}{4} \sin \frac{\varphi}{2}+\left(\frac{1}{4}\right)^{2}\left(\frac{1}{12} \sin \frac{\varphi}{2}-\frac{3}{32} \sin \frac{3 \varphi}{2}\right)\right. \\
& \left.+\left(\frac{1}{4}\right)^{3}\left(\frac{1}{16} \sin \frac{\varphi}{2}-\frac{1}{30} \sin \frac{3 \varphi}{2}+\frac{5}{128} \sin \frac{5 \varphi}{2}\right)\right]
\end{aligned}
$$

with homogeneous Neumann boundary conditions on the crack face. Because the problem is axisymmetric we construct a two-dimensional axi-symmetric finite element (FE) model and solve the Laplace equation over the axi-symmetric cross section using a high-order FE analysis. In Figure 4 left the finite element solution $\left(\tau^{F E}\right)$ at polynomial level $p=8$ is shown whereas in Figure 4 right the difference between the analytical and FE solution is shown $\tau-\tau^{F E}$. As may be noticed $\tau-\tau^{F E}$ is three and a half orders of magnitude smaller compared to $\tau$, indicating on the correctness of the derived analytical solution. If only terms up to $(\rho / R)^{2}$ are applied on the boundary of the domain, then the error $\tau-\tau^{F E}$ increases by one order of magnitude as expected.

2.1.2. A specific example problem - penny-shaped crack with axisymmetric loading and homogeneous Dirichlet BCs. Similarly to subsection 2.1.1 we present here the first terms in the asymptotic series solution for a penny-shaped crack, $\varphi_{1}=-\pi, \omega=2 \pi$ in an axisymmetric domain with homogeneous Dirichlet BCs (8). Now the eigen-pairs are given by

\begin{tabular}{c|c|c}
$k$ & $\alpha_{k}$ & $\phi_{k, 0}$ \\
\hline 1 & $\frac{1}{2}$ & $\cos \frac{\varphi}{2}$ \\
2 & 1 & $\sin \varphi$ \\
3 & $\frac{3}{2}$ & $\cos \frac{3 \varphi}{2}$
\end{tabular}

TABLE 2. First three eigen-pairs for a crack with homogeneous Dirichlet BCs. 


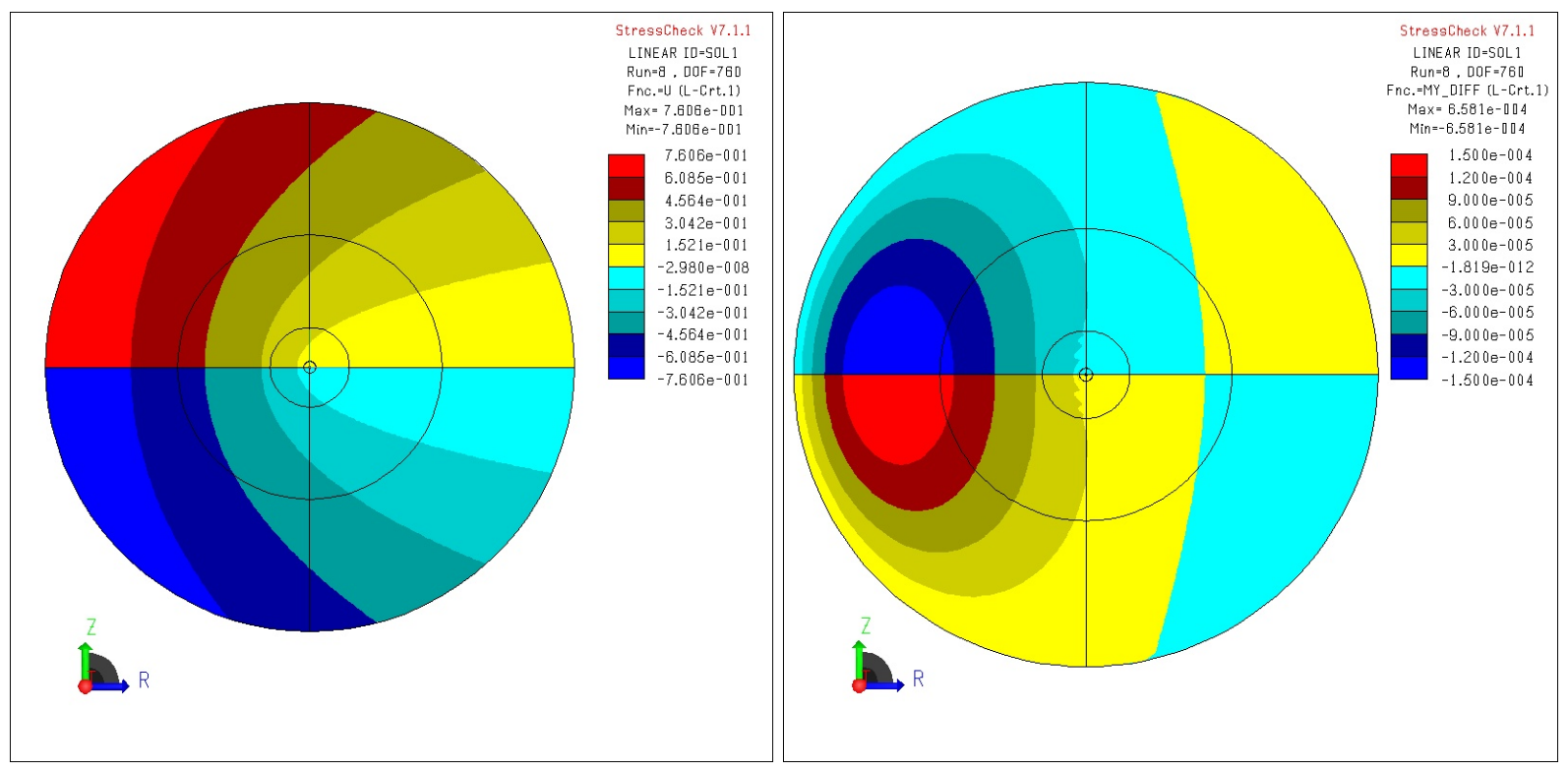

FIGURE 4. Solution (left) and error (right) for the axi-symmetric Laplacian with homogeneous Neumann BCs with $\rho_{\text {out }}=1 / 2, \rho / R=1 / 4$ and $A_{1}=1$ - Series up to $(\rho / R)^{3}$. The axis of symmetry is right to the shown domain with the crack from the center of the circle to the right.

We obtain the following expression for the first terms in the asymptotic series solution:

$$
\begin{array}{rr}
\tau=A_{1} \rho^{\frac{1}{2}}\left[\cos \frac{\varphi}{2}-\left(\frac{\rho}{R}\right) \frac{1}{4} \cos \frac{\varphi}{2}+\left(\frac{\rho}{R}\right)^{2}\left(\frac{1}{12} \cos \frac{\varphi}{2}+\frac{3}{32} \cos \frac{3 \varphi}{2}\right)\right. \\
+\quad & \quad A_{2} \rho \sin \varphi \\
+ & A_{3} \rho^{\frac{3}{2}}\left[\cos \frac{3 \varphi}{2}-\left(\frac{\rho}{R}\right) \frac{1}{4} \cos \frac{\varphi}{2}+\left(\frac{\rho}{R}\right)^{2}\left(\frac{3}{32} \cos \frac{\varphi}{2}+\frac{1}{10} \cos \frac{3 \varphi}{2}-\frac{5}{128} \cos \frac{5 \varphi}{2}\right)+\cdots\right] \\
+ & \left.\quad+\left(\frac{\rho}{R}\right)^{3}\left(-\frac{3}{40} \cos \frac{\varphi}{2}-\frac{5}{128} \cos \frac{3 \varphi}{2}-\frac{3}{70} \cos \frac{5 \varphi}{2}\right)+\cdots\right]
\end{array}
$$

Here we still enforce the orthogonality conditions (20) in order to have uniqueness.

The first terms in the asymptotic solution for the same specific problem are provided in [10, p. 293], where the angular coordinate is measured from the crack face, denoted by $\widetilde{\varphi} \in(0,2 \pi)$, and $R=1$ :

$$
\widetilde{\tau}=c_{1} \rho^{\frac{1}{2}}\left[\sin \frac{\widetilde{\varphi}}{2}-\frac{\rho}{4} \sin \frac{\widetilde{\varphi}}{2}-\frac{\rho^{2}}{32} \sin \frac{3 \widetilde{\varphi}}{2}+\cdots\right] .
$$

If we replace $\widetilde{\varphi}$ by $\varphi+\pi$ and $c_{1}$ by $-A_{1}$, one obtains:

$$
\widetilde{\tau}=A_{1} \rho^{\frac{1}{2}}\left[\cos \frac{\varphi}{2}-\frac{\rho}{4} \cos \frac{\varphi}{2}+\frac{\rho^{2}}{32} \cos \frac{3 \varphi}{2}+\cdots\right]
$$

whereas our formula (46) is (with $R=1$ )

$$
\tau=A_{1} \rho^{\frac{1}{2}}\left[\cos \frac{\varphi}{2}-\frac{\rho}{4} \cos \frac{\varphi}{2}+\rho^{2}\left(\frac{1}{12} \cos \frac{\varphi}{2}+\frac{3}{32} \cos \frac{3 \varphi}{2}\right)+\cdots\right]
$$

So we note a discrepancy between (22) and (23) at the level of the third term in the asymptotics. The source of this error in [10] is a typo in Proposition 1 where instead of $\frac{3}{32}$ the term $\frac{1}{32}$ appears. In 
the proof of this proposition, the right expression is given in equation (32) in [10], however again in the example at the end of that paper the term $\frac{1}{12} \sin \frac{\varphi}{2}$ was forgotten. To verify numerically these formulas, we considered a circular crack in the same torus as in example problem in subsection 2.1.1 with $R=1, \rho_{\text {out }}=1 / 2$ and constructed an axisymmetric FE model. Taking $A_{1}=1$ and $A_{i}=0$, $i=2,3, \cdots$, we prescribed on the outer domain boundary condition either according to (22) or (23) with one term (up to $\rho^{\frac{1}{2}}$ ), two terms (up two $\rho^{3 / 2}$ ) or three terms (up two $\rho^{5 / 2}$ ). We then computed the discrete $L^{2}$ norm of the relative difference between the FE solution and the anticipated "exact solution". Of course that both (22) and (23) provide the same results if up to two terms in the expansion are considered (which are the same), and the relative difference with three terms is different.

The relative difference is defined as:

with

$$
\|e\|_{L^{2}}^{2}=\left[2 \pi \int_{-\pi}^{\pi} \int_{0}^{\rho_{\text {out }}}\left|\tau-\tau_{F E}\right|^{2} \times \rho(R+\rho \cos \varphi) d \rho d \varphi\right] /\|\tau\|_{L^{2}}^{2}
$$

$$
\|\tau\|_{L^{2}}^{2}=2 \pi \int_{-\pi}^{\pi} \int_{0}^{\rho_{\text {out }}}|\tau|^{2} \times \rho(R+\rho \cos \varphi) d \rho d \varphi
$$

The FE solution converged to an estimated $0.2 \%$ relative error in energy norm, the and integration was performed numerically using 90 Gauss point. The obtained relative errors are shown in Table 3.

\begin{tabular}{l|c|c|c|c} 
number of terms & $\|e\|_{L^{2}}^{2}$ & $\|\tau\|_{L^{2}}^{2}$ & $\|\widetilde{e}\|_{L^{2}}^{2}$ & $\|\widetilde{\tau}\|_{L^{2}}^{2}$ \\
\hline 1 & $1.2 \times 10^{-3}$ & $1.554 \times 10^{-1}$ & $1.2 \times 10^{-3}$ & $1.554 \times 10^{-1}$ \\
2 & $9.5 \times 10^{-5}$ & $1.274 \times 10^{-1}$ & $9.5 \times 10^{-5}$ & $1.274 \times 10^{-1}$ \\
3 & $1.77 \times 10^{-5}$ & $1.317 \times 10^{-1}$ & $7.74 \times 10^{-5}$ & $1.277 \times 10^{-1}$
\end{tabular}

TABLE 3. $L^{2}$ relative error - verification of our solution compared to [10].

Inspecting the values in Table 3 one may notice that the relative error of our solution decreases by a factor of $\approx 4$ when adding the third term, compared to a factor of less than 1.2 for the solution in [10], indicating that (23) is the right solution.

2.1.3. A specific example problem - circumferential crack with axisymmetric loading and homogeneous Neumann BCs. Similarly to subsection 2.1 .1 we present herein the first terms in the asymptotic series solution for a circumferential crack, (see Figure 2 (d)) $\varphi_{1}=-\frac{\pi}{2}, \omega=2 \pi$ in an axisymmetric domain with homogeneous Neumann BCs (9), still taking the orthogonality condition (20) into account.

$$
\begin{aligned}
\tau=A_{0}+A_{1} \rho^{\frac{1}{2}}\left[\left(\sin \frac{\varphi}{2}-\right.\right. & \left.\cos \frac{\varphi}{2}\right) \\
& \left.+\left(\frac{\rho}{R}\right)\left(\frac{1}{4}\left(\sin \frac{\varphi}{2}+\cos \frac{\varphi}{2}\right)+\frac{1}{12}\left(\sin \frac{3 \varphi}{2}-\cos \frac{3 \varphi}{2}\right)\right)+\cdots\right]
\end{aligned}
$$

Note that using the modified angular variable $\widetilde{\varphi}:=\varphi-\frac{\pi}{2} \in(-\pi, \pi)$ we obtain the expression

$$
\tau=A_{0}+A_{1} \rho^{\frac{1}{2}}\left[\sin \frac{\widetilde{\varphi}}{2}+\left(\frac{\rho}{R}\right)\left(\frac{1}{4} \cos \frac{\widetilde{\varphi}}{2}+\frac{1}{12} \cos \frac{3 \widetilde{\varphi}}{2}\right)+\cdots\right]
$$

which can be compared with (19).

More generally, if $\omega=2 \pi$, using the angular variable $\widetilde{\varphi}:=\varphi-\varphi_{1}-\pi \in(-\pi, \pi)$ we obtain

$$
\begin{aligned}
\tau=A_{0}+A_{1} \rho^{\frac{1}{2}}\left[\sin \frac{\widetilde{\varphi}}{2}\right. & \\
& \left.-\left(\frac{\rho}{R}\right)\left(\frac{1}{4} \cos \varphi_{1} \sin \frac{\widetilde{\varphi}}{2}+\frac{1}{4} \sin \varphi_{1} \cos \frac{\widetilde{\varphi}}{2}+\frac{1}{12} \sin \varphi_{1} \cos \frac{3 \widetilde{\varphi}}{2}\right)+\cdots\right]
\end{aligned}
$$


As particular cases $\left(\varphi_{1}=-\pi\right.$ and $\left.\varphi_{1}=0\right)$ we find the penny-shaped and the outer circular crack, for which formula (26) coincides with the formula given in [1]:

$$
K_{1}(s) \rho^{\frac{1}{2}}\left(\sin \frac{\widetilde{\varphi}}{2}+\frac{1}{4} \kappa(s) \rho \sin \frac{\widetilde{\varphi}}{2}+\cdots\right)
$$

since in the first case $\kappa(s)=\frac{1}{R}$ and in the second case $\kappa(s)=-\frac{1}{R}$.

2.2. General case. If no axi-symmetric assumption is imposed on the data (only the edge is circular) then the full Laplace operator $\triangle^{3 D}$ in (3) has to be considered. Like for (5)-(6), we find that solutions $\tau$ of (1) are equivalently the solutions of $\left(\frac{r}{R}\right)^{2} \rho^{2} \triangle^{3 D} \tau=0$, i.e.

$$
\begin{aligned}
\left(1+\frac{\rho}{R} \cos \varphi\right)^{2}\left[\left(\rho \partial_{\rho}\right)^{2}+\partial_{\varphi \varphi}\right] & \tau \\
+ & \frac{\rho}{R}\left(1+\frac{\rho}{R} \cos \varphi\right)\left[\cos \varphi\left(\rho \partial_{\rho}\right)-\sin \varphi \partial_{\varphi}\right] \tau+\left(\frac{\rho}{R}\right)^{2} \partial_{\theta \theta} \tau=0 .
\end{aligned}
$$

To condense formulas, let us introduce the operators

$$
m_{0}\left(\rho \partial_{\rho} ; \partial_{\varphi}\right)=\left(\rho \partial_{\rho}\right)^{2}+\partial_{\varphi \varphi}, \quad m_{01}\left(\rho \partial_{\rho} ; \partial_{\varphi}\right)=\cos \varphi\left(\rho \partial_{\rho}\right)-\sin \varphi \partial_{\varphi}
$$

Then equation (28) is equivalent to

$$
m_{0} \tau+\frac{\rho}{R}\left[2 \cos \varphi m_{0}+m_{01}\right] \tau+\left(\frac{\rho}{R}\right)^{2}\left[\cos ^{2} \varphi m_{0}+\cos \varphi m_{01}+\partial_{\theta \theta}\right] \tau=0
$$

In the general case for a circular edge the following form of expansion series is appropriate:

$$
\tau=\sum_{\ell=0,2,4, \ldots} \sum_{k=0} \partial_{\theta}^{\ell} A_{k}(\theta) \rho^{\alpha_{k}}\left(\frac{\rho}{R}\right)^{\ell} \sum_{i=0}^{\infty}\left(\frac{\rho}{R}\right)^{i} \phi_{\ell, k, i}(\varphi)
$$

Remark 5. Notice that $\phi_{0, k, i}=\phi_{k, i}$ (associated with the curvature for an axisymmetric case), so these are known for the axi-symmetric analysis.

Comparing this asymptotic expansion to the case of a straight edge [3], one notices one extra sum, implying that for each primal eigen-function there are two levels of shadow-functions - one set is associated with the derivatives of $A_{k}$ (the index $\ell$ ), and the other set associated with the "curvature terms", i.e. the powers $\rho / R$ (index $i$ ).

The splitting in (30) provides an elegant and convenient way to the formulation of the series expansion of the solution. Introducing the definition for a general term in the expansion (31):

$$
\Phi_{\ell, k, i} \stackrel{\text { def }}{=} \rho^{\alpha_{k}}\left(\frac{\rho}{R}\right)^{\ell+i} \phi_{\ell, k, i}(\varphi)
$$

we observe that

$$
\begin{aligned}
m_{0}\left(\rho \partial_{\rho} ; \partial_{\varphi}\right) \Phi_{\ell, k, i}(\rho, \varphi) & =\rho^{\alpha_{k}}\left(\frac{\rho}{R}\right)^{\ell+i} m_{0}\left(\alpha_{k}+\ell+i ; \partial_{\varphi}\right) \phi_{\ell, k, i}(\varphi), \\
m_{01}\left(\rho \partial_{\rho} ; \partial_{\varphi}\right) \Phi_{\ell, k, i}(\rho, \varphi) & =\rho^{\alpha_{k}}\left(\frac{\rho}{R}\right)^{\ell+i} m_{01}\left(\alpha_{k}+\ell+i ; \partial_{\varphi}\right) \phi_{\ell, k, i}(\varphi),
\end{aligned}
$$


Substituting (31) into (30) one deduces:

$$
\begin{aligned}
0=A_{k}(\theta) \times\left\{m_{0}\left(\alpha_{k}\right) \phi_{0, k, 0}\right. \\
+\left(\frac{\rho}{R}\right)\left[m_{0}\left(\alpha_{k}+1\right) \phi_{0, k, 1}+\left(2 \cos \varphi m_{0}\left(\alpha_{k}\right)+m_{01}\left(\alpha_{k}\right)\right) \phi_{0, k, 0}\right] \\
+\left(\frac{\rho}{R}\right)^{2}\left[m_{0}\left(\alpha_{k}+2\right) \phi_{0, k, 2}+\left(2 \cos \varphi m_{0}\left(\alpha_{k}+1\right)+m_{01}\left(\alpha_{k}+1\right)\right) \phi_{0, k, 1}\right. \\
\left.\left.+\left(\cos ^{2} \varphi m_{0}\left(\alpha_{k}\right)+\cos \varphi m_{01}\left(\alpha_{k}\right)\right) \phi_{0, k, 0}\right]+\cdots\right\} \\
+\quad A_{k}^{\prime \prime}(\theta) \times\left\{\left(\frac{\rho}{R}\right)^{2}\left[m_{0}\left(\alpha_{k}+2\right) \phi_{2, k, 0}+\phi_{0, k, 0}\right]\right. \\
+\left(\frac{\rho}{R}\right)^{3}\left[m_{0}\left(\alpha_{k}+3\right) \phi_{2, k, 1}+\left(2 \cos \varphi m_{0}\left(\alpha_{k}+2\right)+m_{01}\left(\alpha_{k}+2\right)\right) \phi_{2, k, 0}+\phi_{0, k, 1}\right] \\
+\left(\frac{\rho}{R}\right)^{4}\left[m_{0}\left(\alpha_{k}+4\right) \phi_{2, k, 2}+\left(2 \cos \varphi m_{0}\left(\alpha_{k}+3\right)+m_{01}\left(\alpha_{k}+3\right)\right) \phi_{2, k, 1}\right. \\
\left.\left.\quad+\left(\cos ^{2} \varphi m_{0}\left(\alpha_{k}+2\right)+\cos \varphi m_{01}\left(\alpha_{k}+2\right)\right) \phi_{2, k, 0}+\phi_{0, k, 2}\right]+\cdots\right\}
\end{aligned}
$$

Equation (34) has to hold true for any $(\rho / R)^{i}$, and for any $\partial_{\theta}^{\ell} A_{k}$, resulting in the following recursive set of ordinary differential equations for the determination of the eigen-functions and shadows $\phi_{\ell, k, i}(\varphi)$ :

$$
\begin{aligned}
m_{0}\left(\alpha_{k}+\ell+i\right) \phi_{\ell, k, i} & = \\
& -\left(2 \cos \varphi m_{0}\left(\alpha_{k}+\ell+i-1\right)+m_{01}\left(\alpha_{k}+\ell+i-1\right)\right) \phi_{\ell, k, i-1} \\
& -\left(\cos ^{2} \varphi m_{0}\left(\alpha_{k}+\ell+i-2\right)+\cos \varphi m_{01}\left(\alpha_{k}+\ell+i-2\right)\right) \phi_{\ell, k, i-2} \\
& -\phi_{\ell-2, k, i}, \quad \text { for } \quad \ell=0,2,4,6, \cdots, \quad \text { and } \quad i \geq 0 .
\end{aligned}
$$

Here, by convention, $\phi$ 's with negative indices are zero.

Equations (35) for $\ell=0$ are equivalent to equations (15-17) associated with the axi-symmetric case, and for $\ell=2,4,6, \cdots$ results in (36) associated with the non-axi-symmetric case.

$$
\begin{aligned}
& \ell=0 \\
& \text { Equations (15)-(17) for the axi-symmetric case hold. } \\
& \begin{aligned}
\ell= & 2,4,6 \cdots, \quad i \geq 0 \\
\left(\alpha_{k}+i+\ell\right)^{2} \phi_{\ell, k, i}+\phi_{\ell, k, i}^{\prime \prime} & \left.=-\left(\ell+i+\alpha_{k}-1\right)\left[2\left(\ell+i+\alpha_{k}\right)-1\right] \cos \varphi \phi_{\ell, k,(i-1)}\right) \\
& +\sin \varphi \phi_{\ell, k,(i-1)}^{\prime}-2 \cos \varphi \phi_{\ell, k,(i-1)}^{\prime \prime} \\
& -\left(\ell+\alpha_{k}+i-2\right)\left(\ell+\alpha_{k}+i-1\right) \cos ^{2} \varphi \phi_{\ell, k,(i-2)} \\
& +\cos \varphi \sin \varphi \phi_{\ell, k,(i-2)}^{\prime}-\cos ^{2} \varphi \phi_{\ell, k,(i-2)}^{\prime \prime}-\phi_{(\ell-2), k, i}
\end{aligned}
\end{aligned}
$$

Equations (36) are complemented by the homogeneous Dirichlet or Neumann boundary conditions:

$$
\begin{array}{rlrlrl}
\phi_{\ell, k, i}(\varphi) & =0, & \left(\varphi=\varphi_{1}, \varphi_{1}+\omega\right) & & \text { in case of Drichlet BCs } \\
\left(\phi_{\ell, k, i}\right)^{\prime}(\varphi) & =0, & & \left(\varphi=\varphi_{1}, \varphi_{1}+\omega\right) & & \text { in case of Neumann BCs }
\end{array}
$$

2.2.1. A specific example problem - penny-shaped crack for a non-axisymmetric loading and homogeneous Neumann BCs. Again we consider as an example problem a penny-shaped crack $\varphi_{1}=-\pi$, $\omega=2 \pi$, however, the loading may by non-axisymmetric, as well as the outer boundary of the 3-D domain of interest. The eigen-functions and a part of the shadow-functions, $\phi_{0, k, i}(\varphi)$ have been provided 
by (19).As an example, the solution of $\phi_{2,1,0}(\varphi) \quad(\ell=2, k=1, i=0)$ may be obtained from (36), for $k=1 \quad \alpha_{1}=1 / 2$ and $i=0, \ell=2$. All $\phi \mathrm{s}$ with negative indices in the RHS vanish except one:

$$
\left(\frac{1}{2}+0+2\right)^{2} \phi_{2,1,0}+\phi_{2,1,0}^{\prime \prime}=-\phi_{0,1,0},
$$

and the homogeneous Neumann BCs read:

$$
\phi_{2,1,0}^{\prime}(\varphi= \pm \pi)=0 .
$$

From (19), $\phi_{0,1,0}=\sin \frac{\varphi}{2}$, thus the solution of (39) can be taken as the particular solution alone:

$$
\phi_{2,1,0}=-\frac{1}{6} \sin \frac{\varphi}{2}
$$

Once $\phi_{2,1,0}$ is available one may proceed to the computation of $\phi_{2,1,1}(\varphi) \quad(\ell=2, k=1, i=1)$ obtained from (36), for $k=1 \quad \alpha_{1}=1 / 2$ and $i=1, \ell=2$ :

$$
\begin{aligned}
\left(\frac{1}{2}+1+2\right)^{2} \phi_{2,1,1}+\phi_{2,1,1}^{\prime \prime}=- & \left(2+1+\frac{1}{2}-1\right)\left[2\left(2+1+\frac{1}{2}\right)-1\right] \cos \varphi \phi_{2,1,0} \\
& +\sin \varphi \phi_{2,1,0}^{\prime}-2 \cos \varphi \phi_{0,1,0}^{\prime \prime}-\phi_{0,1,1}
\end{aligned}
$$

Substituting $\phi_{2,1,0}$ from (40) and $\phi_{0,1,1}=\frac{1}{4} \sin \frac{\varphi}{2}$ from (19), the particular solution to (41) that satisfies the homogeneous Neumann BCs is:

$$
\phi_{2,1,1}=-\frac{1}{8} \sin \frac{\varphi}{2}+\frac{7}{60} \sin \frac{3 \varphi}{2}
$$

This procedure may be continued, to finally obtain the terms in the series expansion:

$$
\begin{aligned}
\tau= & A_{0}(\theta) \\
+ & A_{0}^{\prime \prime}(\theta)\left(\frac{\rho}{R}\right)^{2}\left[-\frac{1}{4}+\left(\frac{\rho}{R}\right) \frac{5}{16} \cos \varphi-\left(\frac{\rho}{R}\right)^{2}\left(\frac{19}{128}+\frac{11}{64} \cos 2 \varphi\right)+\cdots\right]+\cdots \\
+ & A_{1}(\theta) \rho^{\frac{1}{2}}\left[\sin \frac{\varphi}{2}+\left(\frac{\rho}{R}\right) \frac{1}{4} \sin \frac{\varphi}{2}+\left(\frac{\rho}{R}\right)^{2}\left(\frac{1}{12} \sin \frac{\varphi}{2}-\frac{3}{32} \sin \frac{3 \varphi}{2}\right)+\right. \\
& \left.+\left(\frac{\rho}{R}\right)^{3}\left(\frac{1}{16} \sin \frac{\varphi}{2}-\frac{1}{30} \sin \frac{3 \varphi}{2}+\frac{5}{128} \sin \frac{5 \varphi}{2}\right)+\cdots\right] \\
+ & A_{1}^{\prime \prime}(\theta) \rho^{\frac{1}{2}}\left(\frac{\rho}{R}\right)^{2}\left[-\frac{1}{6} \sin \frac{\varphi}{2}+\left(-\frac{1}{8} \sin \frac{\varphi}{2}+\frac{7}{60} \sin \frac{3 \varphi}{2}\right)\left(\frac{\rho}{R}\right)+\cdots\right]+\cdots
\end{aligned}
$$

Again, the factors corresponding to $A_{0}$ (and all terms of even order) and their derivatives are polynomial in $\left(z_{1}, z_{2}\right)$.

Remark 6. In the vicinity of a crack with a straight edge along the axis $z_{3}$ the solution admits the expansion:

$$
\begin{aligned}
\tau= & A_{0}\left(z_{3}\right)+A_{0}^{\prime \prime}\left(z_{3}\right) r^{2}\left(-\frac{1}{4}\right)+\cdots \\
& +A_{1}\left(z_{3}\right) r^{\frac{1}{2}} \sin \frac{\varphi}{2}+A_{1}^{\prime \prime}\left(z_{3}\right) r^{\frac{5}{2}}\left(-\frac{1}{6} \sin \frac{\varphi}{2}\right)+\cdots
\end{aligned}
$$

One may notice that (44) is composed of the same leading terms associated with $i=0$ as in the expansion (43), as expected.

To assess the correctness of the solution (43) we consider a torus with an inner radius $r_{1}=9$ and an outer radius $r_{2}=11$ having a circular crack with the tip at $R=10$ shown in Figure 3 . This time we apply non-axisymmetric boundary conditions, so a fully 3-D FE model is constructed as presented in Figure 5. Taking $A_{1}=10 \cos \theta$ and $A_{k}=0, k \neq 1$, (notice that $\rho / R=1 / 10$ in the considered 

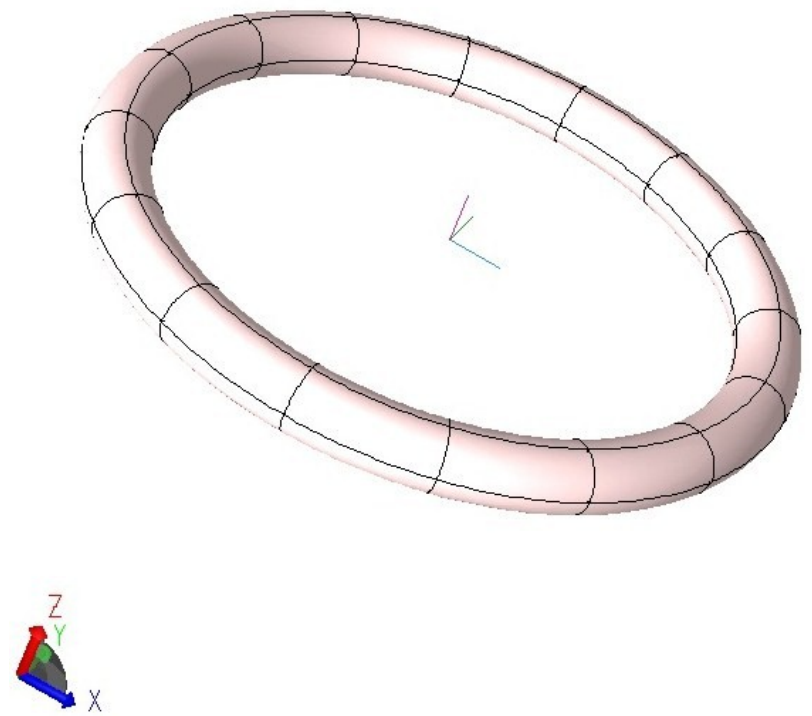

FIGURE 5. The 3-D FE for the torus with a circular crack.

example) we prescribed on the outer surface of the torus Dirichlet boundary conditions according to (43):

$$
\begin{aligned}
\tau= & 10 \cos \theta \sqrt{\frac{1}{10}}\left[\sin \left(\frac{\varphi}{2}\right)+\frac{1}{4} \sin \frac{\varphi}{2}\left(\frac{1}{10}\right)+\left(\frac{1}{12} \sin \frac{\varphi}{2}-\frac{3}{32} \sin \frac{3 \varphi}{2}\right)\left(\frac{1}{10}\right)^{2}+\right. \\
& \left.\left(\frac{1}{16} \sin \frac{\varphi}{2}-\frac{1}{30} \sin \frac{3 \varphi}{2}+\frac{5}{128} \sin \frac{5 \varphi}{2}\right)\left(\frac{1}{10}\right)^{3}\right] \\
& -10 \cos \theta \sqrt{\frac{1}{10}}\left[-\frac{1}{6} \sin \frac{\varphi}{2}\left(\frac{1}{10}\right)^{2}+\left(-\frac{1}{8} \sin \frac{\varphi}{2}+\frac{7}{60} \sin \frac{3 \varphi}{2}\right)\left(\frac{1}{10}\right)^{3}\right]
\end{aligned}
$$

with homogeneous Neumann boundary conditions on the crack face. In Figure 6 left the finite element solution ( $\tau^{F E}$ ) at polynomial level $p=8$ is shown whereas in the right the difference between the analytical and FE solution is shown. As may be noticed $\tau-\tau^{F E}$ is three orders of magnitude smaller compared to $\tau$, assuring the correctness of the derived analytical solution.

2.2.2. A specific example problem - penny-shaped crack for a non-axisymmetric loading and homogeneous Dirichlet BCs. As an example problem the first terms of the asymptotic solution for a pennyshaped crack $\varphi_{1}=-\pi, \omega=2 \pi$ with homogeneous Dirichlet boundary conditions is provided:

$$
\begin{aligned}
\tau= & A_{1}(\theta) \rho^{\frac{1}{2}}\left[\cos \frac{\varphi}{2}-\frac{1}{4} \cos \frac{\varphi}{2}\left(\frac{\rho}{R}\right)+\left(\frac{1}{12} \cos \frac{\varphi}{2}+\frac{3}{32} \cos \frac{3 \varphi}{2}\right)\left(\frac{\rho}{R}\right)^{2}+\right. \\
& \left.\left(-\frac{1}{16} \cos \frac{\varphi}{2}-\frac{1}{30} \cos \frac{3 \varphi}{2}-\frac{5}{128} \cos \frac{5 \varphi}{2}\right)\left(\frac{\rho}{R}\right)^{3}+\cdots\right] \\
+ & A_{1}^{\prime \prime}(\theta) \rho^{\frac{1}{2}}\left(\frac{\rho}{R}\right)^{2}\left[-\frac{1}{6} \cos \frac{\varphi}{2}+\left(\frac{1}{8} \cos \frac{\varphi}{2}+\frac{7}{60} \cos \frac{3 \varphi}{2}\right)\left(\frac{\rho}{R}\right)+\cdots\right] \\
+ & \cdots
\end{aligned}
$$

2.2.3. A specific example problem - hollow cylinder with non-axisymmetric loading and homogeneous Neumann BCs. Consider the circular edge having a solid angle of $3 \pi / 2$ as the upper corner in Figure 2 


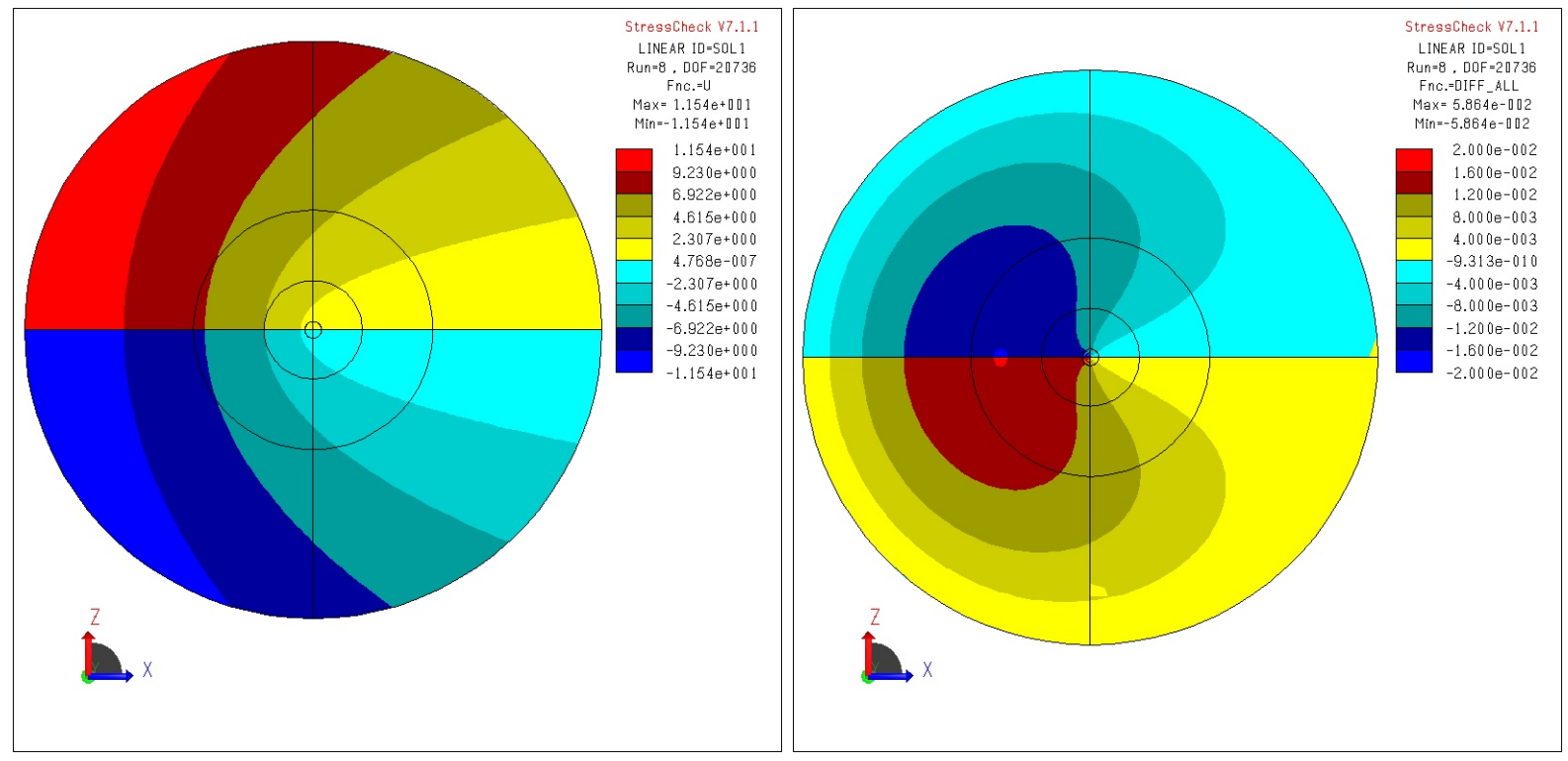

FIGURE 6. The solution $\tau_{F E}$ on a typical cross-section $(\theta=0)$ of the 3-D torus (Left) and the difference between the analytical and FE solution on same cross-section (Right). The axis of symmetry is right to the shown domain with the crack from the center of the circle to the right.

(a) with homogeneous Neumann BCs. In this case $\varphi \in\left(-\frac{\pi}{2}, \pi\right), \alpha_{0}=0$ and $\alpha_{1}=2 / 3, \alpha_{4}=\alpha_{5}=$ $\alpha_{6}=1 \cdots$, and the first few terms in the asymptotic solution are given by:

$$
\begin{aligned}
\tau=A_{0}+A_{0}^{\prime \prime} & \left(\frac{\rho}{R}\right)^{2}\left(-\frac{1}{4}\right) \\
+A_{1} \rho^{2 / 3} & {\left[\sin \frac{2 \varphi}{3}-\frac{1}{\sqrt{3}} \cos \frac{2 \varphi}{3}\right.} \\
& +\left(\frac{\rho}{R}\right) \frac{1}{60}\left(5 \sqrt{3} \cos \frac{\varphi}{3}-\sqrt{3} \cos \frac{5 \varphi}{3}+15 \sin \frac{\varphi}{3}+3 \sin \frac{5 \varphi}{3}\right) \\
& \left.+\left(\frac{\rho}{R}\right)^{2} \frac{1}{160}\left(12 \sin \frac{2 \varphi}{3}-4 \sqrt{3} \cos \frac{2 \varphi}{3}-15 \sin \frac{4 \varphi}{3}-5 \sqrt{3} \cos \frac{4 \varphi}{3}\right)\right]+ \\
+ & A_{1}^{\prime \prime} \rho^{2 / 3}\left(\frac{\rho}{R}\right)^{2}\left[\frac{1}{20}\left(\sqrt{3} \cos \frac{2 \varphi}{3}-3 \sin \frac{2 \varphi}{3}\right)\right]+\cdots
\end{aligned}
$$

2.2.4. A specific example problem - exterior circular crack with non-axisymmetric loading and homogeneous Neumann BCs. Consider the circular external crack as in Figure 2 (b) with homogeneous Neumann BCs. In this case $\varphi \in(0,2 \pi)$ and the first few terms in the asymptotic solution are given by:

$$
\begin{aligned}
\tau= & A_{0}+A_{0}^{\prime \prime}\left(\frac{\rho}{R}\right)^{2}\left(-\frac{1}{4}\right) \\
& +A_{1} \rho^{1 / 2}\left[\cos \frac{\varphi}{2}-\left(\frac{\rho}{R}\right) \frac{1}{4} \cos \frac{\varphi}{2}+\left(\frac{\rho}{R}\right)^{2}\left(\frac{1}{12} \cos \frac{\varphi}{2}+\frac{3}{32} \cos \frac{3 \varphi}{2}\right)\right] \\
& +A_{1}^{\prime \prime} \rho^{1 / 2}\left(\frac{\rho}{R}\right)^{2}\left[-\frac{1}{6} \cos \frac{\varphi}{2}\right]+\cdots
\end{aligned}
$$




\section{ASYMPTOTIC SOLUTION FOR THE ELASTICITY SYSTEM}

The point of departure for the elasticity system is the three equilibrium equations given in a Cartesian system by:

$$
\sum_{i=1}^{3} \frac{\partial \sigma_{i j}}{\partial x_{i}}+F_{j}=0, \quad j=1,2,3,
$$

where $\sigma_{i j}$ are the components of the Cartesian stress tensor, and $F_{j}$ are the body forces. For vanishing body forces in the vicinity of the singular edge, i.e. $F_{1}=F_{2}=F_{3}=0$ we reformulate the equilibrium equations to be expressed in the coordinate system $\rho, \varphi, \theta$ shown in Figure 1:

$$
x_{1}=(R+\rho \cos \varphi) \cos \theta, \quad x_{2}=(R+\rho \cos \varphi) \sin \theta, \quad x_{3}=\rho \sin \varphi
$$

The Cartesian components of the displacements $\left(u_{1}, u_{2}, u_{3}\right)^{\top}$ are connected to the local coordinate system displacements $\left(u_{\rho}, u_{\varphi}, u_{\theta}\right)^{\top}$ by:

$$
\begin{aligned}
& u_{1}=\left(u_{\rho} \cos \varphi-u_{\varphi} \sin \varphi\right) \cos \theta-u_{\theta} \sin \theta \\
& u_{2}=\left(u_{\rho} \cos \varphi-u_{\varphi} \sin \varphi\right) \sin \theta+u_{\theta} \cos \theta \\
& u_{3}=u_{\rho} \sin \varphi+u_{\varphi} \cos \varphi
\end{aligned}
$$

The kinematic connection between displacements and strains in the coordinate system $\rho, \varphi, \theta$ can be derived using the metric tensor [11]:

$$
\begin{aligned}
\varepsilon_{\rho \rho} & =\frac{\partial u_{\rho}}{\partial \rho} \\
\varepsilon_{\varphi \varphi} & =\frac{1}{\rho} \frac{\partial u_{\varphi}}{\partial \varphi}+\frac{u_{\rho}}{\rho} \\
\varepsilon_{\theta \theta} & =\frac{1}{r}\left(\frac{\partial u_{\theta}}{\partial \theta}+u_{\rho} \cos \varphi-u_{\varphi} \sin \varphi\right) \\
\varepsilon_{\rho \varphi} & =\frac{1}{2}\left[\frac{1}{\rho} \frac{\partial u_{\rho}}{\partial \varphi}+\frac{u_{\varphi}}{\rho}-\frac{u_{\varphi}}{\rho}\right] \\
\varepsilon_{\varphi \theta} & =\frac{1}{2}\left[\frac{1}{r} \frac{\partial u_{\varphi}}{\partial \theta}+\frac{1}{\rho} \frac{\partial u_{\theta}}{\varphi}+\frac{1}{r} u_{\theta} \sin \varphi\right] \\
\varepsilon_{\rho \theta} & =\frac{1}{2}\left[\frac{1}{r} \frac{\partial u_{\rho}}{\partial \theta}+\frac{\partial u_{\theta}}{\rho}-\frac{1}{r} u_{\theta} \cos \varphi\right]
\end{aligned}
$$

These connections are identical to [7, Eq. (3)]. In the new curvilinear coordinate system the equilibrium equations in $\rho, \varphi, \theta$ directions read [11]:

$$
\begin{aligned}
& 0=\frac{\partial \sigma_{\rho \rho}}{\partial \rho}+\frac{1}{\rho} \frac{\partial \sigma_{\rho \varphi}}{\partial \varphi}+\frac{\sigma_{\rho \rho}-\sigma_{\varphi \varphi}}{\rho}+\frac{1}{r}\left(\frac{\partial \sigma_{\rho \theta}}{\partial \theta}+\left(\sigma_{\rho \rho}-\sigma_{\theta \theta}\right) \cos \varphi-\sigma_{\rho \varphi} \sin \varphi\right) \\
& 0=\frac{1}{\rho} \frac{\partial \sigma_{\varphi \varphi}}{\partial \varphi}+\frac{\partial \sigma_{\rho \varphi}}{\partial \rho}+\frac{2}{\rho} \sigma_{\rho \varphi}+\frac{1}{r}\left(\frac{\partial \sigma_{\varphi \theta}}{\partial \theta}+\left(\sigma_{\theta \theta}-\sigma_{\varphi \varphi}\right) \sin \varphi+\sigma_{\rho \varphi} \cos \varphi\right) \\
& 0=\frac{\partial \sigma_{\rho \theta}}{\partial \rho}+\frac{1}{\rho} \sigma_{\rho \theta}+\frac{1}{\rho} \frac{\partial \sigma_{\varphi \theta}}{\partial \varphi}+\frac{1}{r}\left(\frac{\partial \sigma_{\theta \theta}}{\partial \theta}+2 \sigma_{\rho \theta} \cos \varphi-2 \sigma_{\varphi \theta} \sin \varphi\right) .
\end{aligned}
$$

We consider an isotropic elastic homogeneous material with Lamé constants $\lambda$ and $\mu$ (kinematic equations and Hooke's law given by:

$$
\sigma_{i j}=\lambda \delta_{i j} \sum_{k=1}^{3} \varepsilon_{k k}+2 \mu \varepsilon_{i j}, \quad i, j=\rho, \varphi, \theta
$$


The stresses in terms of displacements are given by:

$$
\begin{aligned}
& \left(\begin{array}{c}
\sigma_{\rho \rho} \\
\sigma_{\theta \theta} \\
\sigma_{\varphi \varphi} \\
\sigma_{\rho \theta} \\
\sigma_{\rho \varphi} \\
\sigma_{\theta \varphi}
\end{array}\right)=\left(\begin{array}{ccc}
\lambda \frac{1}{\rho}+(\lambda+2 \mu) \partial_{\rho} & \lambda \frac{1}{\rho} \partial_{\varphi} & 0 \\
\lambda\left(\frac{1}{\rho}+\partial_{\rho}\right) & \lambda \frac{1}{\rho} \partial_{\varphi} & 0 \\
(\lambda+2 \mu) \frac{1}{\rho}+\lambda \partial_{\rho} & (\lambda+2 \mu) \frac{1}{\rho} \partial_{\varphi} & 0 \\
0 & 0 & \mu \partial_{\rho} \\
\mu \frac{1}{\rho} \partial_{\varphi} & \mu\left(-\frac{1}{\rho}+\partial_{\rho}\right) & 0 \\
0 & 0 & \mu \frac{1}{\rho} \partial_{\varphi}
\end{array}\right)\left(\begin{array}{c}
u_{\rho} \\
u_{\varphi} \\
u_{\theta}
\end{array}\right) \\
& +\frac{1}{r}\left(\begin{array}{ccc}
\lambda \cos \varphi & -\lambda \sin \varphi & 0 \\
(\lambda+2 \mu) \cos \varphi & -(\lambda+2 \mu) \sin \varphi & 0 \\
\lambda \cos \varphi & -\lambda \sin \varphi & 0 \\
0 & 0 & -\mu \cos \varphi \\
0 & 0 & 0 \\
0 & 0 & \mu \sin \varphi
\end{array}\right)\left(\begin{array}{c}
u_{\rho} \\
u_{\varphi} \\
u_{\theta}
\end{array}\right)+\frac{1}{r}\left(\begin{array}{ccc}
0 & 0 & \lambda \\
0 & 0 & \lambda+2 \mu \\
0 & 0 & \lambda \\
\mu & 0 & 0 \\
0 & 0 & 0 \\
0 & \mu & 0
\end{array}\right) \partial_{\theta}\left(\begin{array}{c}
u_{\rho} \\
u_{\varphi} \\
u_{\theta}
\end{array}\right)
\end{aligned}
$$

Inserting (56) into (52)-(54) and multiplying by $r^{2}(\rho / R)^{2}=\rho^{2}\left(1+\frac{\rho}{R} \cos \varphi\right)^{2}$ one obtains the NavierLamé system:

$$
\begin{aligned}
0= & \left(1+\frac{\rho}{R} \cos \varphi\right)^{2}\left\{(\lambda+2 \mu)\left[\left(\rho \partial_{\rho}\right)^{2}-1\right] u_{\rho}+\mu \partial_{\varphi \varphi} u_{\rho}-(\lambda+3 \mu) \partial_{\varphi} u_{\varphi}+(\lambda+\mu) \partial_{\rho \varphi} u_{\varphi}\right\} \\
& +\left(1+\frac{\rho}{R} \cos \varphi\right) \frac{\rho}{R}\left[(\lambda+2 \mu) \cos \varphi \rho \partial_{\rho} u_{\rho}-(\lambda+\mu) \sin \varphi \rho \partial_{\rho} u_{\varphi}+\mu \sin \varphi\left(u_{\varphi}-\partial_{\varphi} u_{\rho}\right)+(\lambda+\mu) \rho \partial_{\rho \theta} u_{\theta}\right] \\
& +\left(\frac{\rho}{R}\right)^{2}\left[(\lambda+2 \mu) \cos \varphi\left(u_{\varphi} \sin \varphi-u_{\rho} \cos \varphi\right)+\mu \partial_{\theta \theta} u_{\rho}-(\lambda+3 \mu) \cos \varphi \partial_{\theta} u_{\theta}\right] \\
0= & \left(1+\frac{\rho}{R} \cos \varphi\right)^{2}\left\{\mu\left[\left(\rho \partial_{\rho}\right)^{2}-1\right] u_{\varphi}+(\lambda+2 \mu) \partial_{\varphi \varphi} u_{\varphi}+(\lambda+3 \mu) \partial_{\varphi} u_{\rho}+(\lambda+\mu) \rho \partial_{\rho \varphi} u_{\rho}\right\} \\
& +\left(1+\frac{\rho}{R} \cos \varphi\right) \frac{\rho}{R}\left[(\lambda+\mu) \cos \varphi\left(\partial_{\varphi} u_{\rho}-u_{\varphi}\right)+\mu \cos \varphi \rho \partial_{\rho} u_{\varphi}-(\lambda+2 \mu) \sin \varphi\left(\partial_{\varphi} u_{\varphi}+u_{\rho}\right)+(\lambda+\mu) \partial_{\varphi \theta} u_{\theta}\right] \\
& +\left(\frac{\rho}{R}\right)^{2}\left[(\lambda+2 \mu) \sin \varphi\left(u_{\rho} \cos \varphi-u_{\varphi} \sin \varphi\right)+\mu \partial_{\theta \theta} u_{\varphi}+(\lambda+3 \mu) \sin \varphi \partial_{\theta} u_{\theta}\right] \\
0= & \left(1+\frac{\rho}{R} \cos \varphi\right)^{2} \mu\left[\left(\rho \partial_{\rho}\right)^{2}+\partial_{\varphi \varphi}\right] u_{\theta} \\
& +\left(1+\frac{\rho}{R} \cos \varphi\right) \frac{\rho}{R}\left[\mu\left(\cos \varphi \rho \partial_{\rho} u_{\theta}-\sin \varphi \partial_{\varphi} u_{\theta}\right)+(\lambda+\mu)\left(\partial_{\theta} u_{\rho}+\partial_{\varphi \theta} u_{\varphi}+\rho \partial_{\rho \theta} u_{\rho}\right)\right] \\
& +\left(\frac{\rho}{R}\right)^{2}\left[-\mu u_{\theta}+(\lambda+2 \mu) \partial_{\theta \theta} u_{\theta}+(\lambda+3 \mu)\left(\cos \varphi \partial_{\theta} u_{\rho}-\sin \varphi \partial_{\theta} u_{\varphi}\right)\right]
\end{aligned}
$$

The Navier-Lamé equations are complemented by homogeneous boundary conditions on the faces intersecting at the singular edge:

$$
\begin{aligned}
& u_{\rho}=u_{\varphi}=u_{\theta}=0 \quad \text { on } \Gamma_{1} \cup \Gamma_{2} \quad \text { Clamped BCs } \\
& t_{\varphi}=t_{\rho}=t_{\theta}=0 \quad \text { on } \Gamma_{1} \cup \Gamma_{2} \quad \text { Traction Free BCs, }
\end{aligned}
$$

where $t$ is the traction vector on the boundary. On the boundaries $\Gamma_{1}, \Gamma_{2}$, i.e. for $\varphi=\varphi_{1}, \varphi_{2}$, the traction free BCs (61) are expressed in terms of the stresses using Cauchy's law:

$$
\sigma_{\varphi \varphi}=\sigma_{\rho \varphi}=\sigma_{\theta \varphi}=0, \quad \varphi=\varphi_{1}, \varphi_{2}
$$


Denoting the displacement vector by $\boldsymbol{u}=\left(u_{\rho}, u_{\varphi}, u_{\theta}\right)^{\top}$, the Navier-Lamé (57-59) system is split as follows:

$$
\begin{aligned}
& {\left[\left(1+\frac{\rho}{R} \cos \varphi\right)^{2}\left[M_{0}\right]+\left(1+\frac{\rho}{R} \cos \varphi\right)\left(\frac{\rho}{R}\right)\left[M_{01}\right]+\left(\frac{\rho}{R}\right)^{2}\left[M_{02}\right]\right.} \\
& \left.\quad+\left(1+\frac{\rho}{R} \cos \varphi\right)\left(\frac{\rho}{R}\right)\left[M_{10}\right] \partial_{\theta}+\left(\frac{\rho}{R}\right)^{2}\left[M_{11}\right] \partial_{\theta}+\left(\frac{\rho}{R}\right)^{2}\left[M_{2}\right] \partial_{\theta \theta}\right] \boldsymbol{u}=\mathbf{0},
\end{aligned}
$$

with

$$
\left[M_{0}\right]=\left(\begin{array}{ccc}
(\lambda+2 \mu)\left(\left(\rho \partial_{\rho}\right)^{2}-1\right)+\mu \partial_{\varphi \varphi} & -(\lambda+3 \mu) \partial_{\varphi}+(\lambda+\mu) \rho \partial_{\rho \varphi} & 0 \\
(\lambda+\mu) \rho \partial_{\rho \varphi}+(\lambda+3 \mu) \partial_{\varphi} & (\lambda+2 \mu) \partial_{\varphi \varphi}+\mu\left(\left(\rho \partial_{\rho}\right)^{2}-1\right) & 0 \\
0 & 0 & \mu\left(\left(\rho \partial_{\rho}\right)^{2}+\partial_{\varphi \varphi}\right)
\end{array}\right)
$$

$\left[M_{01}\right]=\left(\begin{array}{ccc}(\lambda+2 \mu) \cos \varphi \rho \partial_{\rho}-\mu \sin \varphi \partial_{\varphi} & \sin \varphi\left[-(\lambda+\mu) \rho \partial_{\rho}+\mu\right] & 0 \\ (\lambda+\mu) \cos \varphi \partial_{\varphi}-(\lambda+2 \mu) \sin \varphi & \cos \varphi\left[-(\lambda+\mu)+\mu \rho \partial_{\rho}\right]-(\lambda+2 \mu) \sin \varphi \partial_{\varphi} & 0 \\ 0 & 0 & \mu\left(\cos \varphi \rho \partial_{\rho}-\sin \varphi \partial_{\varphi}\right)\end{array}\right)$

$$
\begin{aligned}
& {\left[M_{02}\right]=\left(\begin{array}{ccc}
-(\lambda+2 \mu) \cos ^{2} \varphi & (\lambda+2 \mu) \sin \varphi \cos \varphi & 0 \\
(\lambda+2 \mu) \sin \varphi \cos \varphi & -(\lambda+2 \mu) \sin ^{2} \varphi & 0 \\
0 & 0 & -\mu
\end{array}\right)} \\
& {\left[M_{10}\right]=\left(\begin{array}{ccc}
0 & 0 & (\lambda+\mu) \rho \partial_{\rho} \\
0 & 0 & (\lambda+\mu) \partial_{\varphi} \\
(\lambda+\mu)\left(\rho \partial_{\rho}+1\right) & (\lambda+\mu) \partial_{\varphi} & 0
\end{array}\right),} \\
& {\left[M_{11}\right]=\left(\begin{array}{ccc}
0 & 0 & -(\lambda+3 \mu) \cos \varphi \\
0 & 0 & (\lambda+3 \mu) \sin \varphi \\
(\lambda+3 \mu) \cos \varphi & -(\lambda+3 \mu) \sin \varphi & 0
\end{array}\right),} \\
& {\left[M_{2}\right]=\left(\begin{array}{ccc}
\mu & 0 & 0 \\
0 & \mu & 0 \\
0 & 0 & (\lambda+2 \mu)
\end{array}\right) .}
\end{aligned}
$$

Following same asymptotic series expansion shown to be appropriate for the Laplace operator, we assume herein an expansion for the displacements of the form:

$$
\boldsymbol{u}=\sum_{\ell=0} \sum_{k=0} \partial_{\theta}^{\ell} A_{k}(\theta) \rho^{\alpha_{k}} \sum_{i=0}^{\infty}\left(\frac{\rho}{R}\right)^{i+\ell}\left\{\begin{array}{l}
\phi_{\rho}(\varphi) \\
\phi_{\varphi}(\varphi) \\
\phi_{\theta}(\varphi)
\end{array}\right\}_{\ell, k, i}=\sum_{\ell=0} \sum_{k=0} \partial_{\theta}^{\ell} A_{k}(\theta) \rho^{\alpha} \sum_{i=0}^{\infty}\left(\frac{\rho}{R}\right)^{i+\ell} \phi_{\ell, k, i}
$$

Comparing this asymptotic expansion to the case of a straight edge, one notices one extra sum, implying that for each primal eigen-function there are two sets of shadow-functions - one set is associated with the derivatives of $A_{k}$, and the other set associated with the "curvature terms", i.e. the powers $\rho / R$.

Inserting (69) in (62) so to gather terms of same order of derivatives of $A_{k}$, and same order of powers $\rho / R$, we obtain the following recursive formula for the computation of the primal and shadow functions:

$$
\begin{aligned}
{\left[m_{0}\right] \boldsymbol{\phi}_{\ell, k, i}=} & -\left(2 \cos \varphi\left[m_{0}\right]+\left[m_{01}\right]\right) \boldsymbol{\phi}_{\ell, k, i-1}-\left(\cos ^{2} \varphi\left[m_{0}\right]+\cos \varphi\left[m_{01}\right]+\left[m_{02}\right]\right) \boldsymbol{\phi}_{\ell, k, i-2} \\
& -\left[m_{10}\right] \boldsymbol{\phi}_{\ell-1, k, i}-\left(\cos \varphi\left[m_{10}\right]+\left[m_{11}\right]\right) \boldsymbol{\phi}_{\ell-1, k, i-1}-\left[m_{2}\right] \boldsymbol{\phi}_{\ell-2, k, i}, \quad \ell \geq 0, i \geq 0
\end{aligned}
$$


where $\phi$ 's with negative indices are set to zero, and

$$
\left[m_{0}\right] \phi_{\ell, k, i}=\left(\begin{array}{ccc}
(\lambda+2 \mu)\left(\beta^{2}-1\right)+\mu \partial_{\varphi \varphi} & ((\lambda+\mu) \beta-(\lambda+3 \mu)) \partial_{\varphi} & 0 \\
((\lambda+\mu) \beta+(\lambda+3 \mu)) \partial_{\varphi} & \mu\left(\beta^{2}-1\right)+(\lambda+2 \mu) \partial_{\varphi \varphi} & 0 \\
0 & 0 & \mu\left(\beta^{2}+\partial_{\varphi \varphi}\right)
\end{array}\right) \phi_{\ell, k, i}
$$

$$
\left[m_{01}\right] \phi_{\ell, k, i}=\left(\begin{array}{ccc}
(\lambda+2 \mu) \cos \varphi \beta-\mu \sin \varphi \partial_{\varphi} & \sin \varphi(\mu-(\lambda+\mu) \beta) & 0 \\
-(\lambda+2 \mu) \sin \varphi+(\lambda+\mu) \cos \varphi \partial_{\varphi} & \cos \varphi(\mu(\beta-1)-\lambda)-(\lambda+2 \mu) \sin \varphi \partial_{\varphi} & 0 \\
0 & 0 & \mu\left(\beta \cos \varphi-\sin \varphi \partial_{\varphi}\right)
\end{array}\right) \boldsymbol{\phi}_{\ell, k, i}
$$

$$
\begin{aligned}
& {\left[m_{02}\right] \phi_{\ell, k, i}=\left(\begin{array}{ccc}
-(\lambda+2 \mu) \cos ^{2} \varphi & (\lambda+2 \mu) \cos \varphi \sin \varphi & 0 \\
(\lambda+2 \mu) \sin \varphi \cos \varphi & -(\lambda+2 \mu) \sin ^{2} \varphi & 0 \\
0 & 0 & -\mu
\end{array}\right) \phi_{\ell, k, i}} \\
& {\left[m_{10}\right] \phi_{\ell, k, i}=\left(\begin{array}{ccc}
0 & 0 & (\lambda+\mu) \beta \\
0 & 0 & (\lambda+\mu) \partial_{\varphi} \\
(\lambda+\mu) \beta & (\lambda+\mu) \partial_{\varphi} & 0
\end{array}\right) \phi_{\ell, k, i}} \\
& {\left[m_{11}\right] \phi_{\ell, k, i}=\left(\begin{array}{ccc}
0 & 0 & -(\lambda+3 \mu) \cos \varphi \\
0 & 0 & (\lambda+3 \mu) \sin \varphi \\
(\lambda+3 \mu) \cos \varphi & -(\lambda+3 \mu) \sin \varphi & 0
\end{array}\right) \phi_{\ell, k, i}} \\
& {\left[m_{2}\right] \phi_{\ell, k, i}=\left(\begin{array}{ccc}
\mu & 0 & 0 \\
0 & \mu & 0 \\
0 & 0 & (\lambda+2 \mu)
\end{array}\right) \phi_{\ell, k, i}}
\end{aligned}
$$

where

$$
\beta=\left(\alpha_{k}+\ell+i\right) .
$$

3.1. Homogeneous boundary conditions. The system of ODEs (70) is complemented by either clamped (homogenous Dirichlet) boundary conditions or traction free (homogeneous Neumann) boundary conditions on $\varphi=\varphi_{1}$ and $\varphi=\varphi_{2}=\varphi_{1}+\omega$. The clamped boundary conditions $\boldsymbol{u}\left(\varphi_{1}\right)=\mathbf{0}, \boldsymbol{u}\left(\varphi_{2}\right)=\mathbf{0}$ imply:

$$
\boldsymbol{\phi}_{\ell, k, i}\left(\varphi_{1}\right)=\boldsymbol{\phi}_{\ell, k, i}\left(\varphi_{2}\right)=\mathbf{0}, \quad \forall \ell, \alpha_{k}, i
$$

The traction free boundary conditions imply:

$$
\left(\sigma_{\varphi \varphi}, \sigma_{\rho \varphi}, \sigma_{\theta \varphi}\right)^{\top}=\mathbf{0}, \quad \varphi=\varphi_{1}, \varphi_{2}
$$

Multiplying (78) by $r \frac{\rho}{R}$ yields:

$$
\left[\left(1+\frac{\rho}{R} \cos \varphi\right)\left[T_{0}\right]+\frac{\rho}{R}\left[T_{01}\right]+\frac{\rho}{R}\left[T_{1}\right] \partial_{\theta}\right] \boldsymbol{u}=\mathbf{0}, \quad \varphi=\varphi_{1}, \varphi_{2}
$$

where,

$$
\begin{gathered}
{\left[T_{0}\right]=\left(\begin{array}{ccc}
(\lambda+2 \mu)+\lambda \rho \partial_{\rho} & (\lambda+2 \mu) \partial_{\varphi} & 0 \\
\mu \partial_{\varphi} & -\mu\left(1-\rho \partial_{\rho}\right) & 0 \\
0 & 0 & \mu \partial_{\varphi}
\end{array}\right)} \\
{\left[T_{01}\right]=\left(\begin{array}{ccc}
\lambda \cos \varphi & -\lambda \sin \varphi & 0 \\
0 & 0 & 0 \\
0 & 0 & \mu \sin \varphi
\end{array}\right), \quad\left[T_{1}\right]=\left(\begin{array}{lll}
0 & 0 & \lambda \\
0 & 0 & 0 \\
0 & \mu & 0
\end{array}\right)}
\end{gathered}
$$

Inserting (69) in (79) we obtain the following boundary conditions on the primal and shadow functions $\forall \ell, \alpha_{k}, i$ : 


$$
\left[t_{0}\right] \phi_{\ell, k, i}=-\left(\cos \varphi\left[t_{0}\right]+\left[t_{01}\right]\right) \phi_{\ell, k, i-1}-\left[t_{1}\right] \phi_{\ell-1, k, i}, \quad \varphi=\varphi_{1}, \varphi_{2}
$$

again $\phi$ 's with negative indices are set to zero, and

$$
\begin{gathered}
{\left[t_{0}\right] \phi_{\ell, k, i}=\left(\begin{array}{ccc}
2 \mu+\lambda(\beta+1) & (\lambda+2 \mu) \partial_{\varphi} & 0 \\
\mu \partial_{\varphi} & \mu(\beta-1) & 0 \\
0 & 0 & \mu \partial_{\varphi}
\end{array}\right) \boldsymbol{\phi}_{\ell, k, i}} \\
{\left[t_{01}\right] \phi_{\ell, k, i}=\left(\begin{array}{ccc}
\lambda \cos \varphi & -\lambda \sin \varphi & 0 \\
0 & 0 & 0 \\
0 & 0 & \mu \sin \varphi
\end{array}\right) \phi_{\ell, k, i}, \quad\left[t_{1}\right] \phi_{\ell, k, i}=\left(\begin{array}{ccc}
0 & 0 & \lambda \\
0 & 0 & 0 \\
0 & \mu & 0
\end{array}\right) \phi_{\ell, k, i}}
\end{gathered}
$$

3.2. Axi-symmetric case. For an axi-symmetric domain and boundary conditions all derivatives with respect to $\theta$ vanish so (62) is simplified to:

$$
\left[\left(1+\frac{\rho}{R} \cos \varphi\right)^{2}\left[M_{0}\right]+\left(1+\frac{\rho}{R} \cos \varphi\right)\left(\frac{\rho}{R}\right)\left[M_{01}\right]+\left(\frac{\rho}{R}\right)^{2}\left[M_{02}\right]\right] \boldsymbol{u}=\mathbf{0},
$$

Notice that third equation in the set (85) is in terms of $u_{\theta}$ alone and decoupled from the first two equations, thus the solution to the displacement $u_{\theta}$ is decoupled from $u_{\rho}$ and $u_{\varphi}$ for the axi-symmetric case.

Remark 7. The equation for determining $u_{\theta}$ is very similar (but not identical) to the Laplace equation (compare to (4)). The difference is the last term $\frac{1}{r^{2}} u_{\theta}$. This term results in a slightly different solution of $u_{\theta}$ compared to the Laplace solution only when inspecting higher shadows ( $i \geq 2$ ).

The asymptotic series expansion in case of an axi-symmetric solution is obtained by taking $\ell=0$ in (69):

$$
\boldsymbol{u}=\sum_{k=0}^{\infty} A_{k} \rho^{\alpha_{k}} \sum_{i=0}^{\infty}\left(\frac{\rho}{R}\right)^{i}\left\{\begin{array}{l}
\phi_{\rho}(\varphi) \\
\phi_{\varphi}(\varphi) \\
\phi_{\theta}(\varphi)
\end{array}\right\}_{0, k, i}
$$

In the axi-symmetric case, $\ell \equiv 0$, therefore the recursive formulas (70) and (82) are simplified to:

$$
\begin{aligned}
{\left[m_{0}\right] \boldsymbol{\phi}_{0, k, i}=} & -\left(2 \cos \varphi\left[m_{0}\right]+\left[m_{01}\right]\right) \boldsymbol{\phi}_{0, k, i-1} \\
& -\left(\cos ^{2} \varphi\left[m_{0}\right]+\cos \varphi\left[m_{01}\right]+\left[m_{02}\right]\right) \phi_{0, k, i-2} \quad i \geq 0
\end{aligned}
$$

and

$$
\left[t_{0}\right] \phi_{0, k, i}=-\left(\cos \varphi\left[t_{0}\right]+\left[t_{01}\right]\right) \phi_{0, k, i-1}, \quad \varphi=\varphi_{1}, \varphi_{2}
$$

3.2.1. A specific example problem - penny-shaped crack with axisymmetric loading and traction free $B C$ s. In the case of a penny shaped crack $\omega=2 \pi, \alpha_{1}=\alpha_{2}=\alpha_{3}=0$ and $\alpha_{4}=\alpha_{5}=\alpha_{6}=\frac{1}{2}$, the expressions for the traction free boundary condition (87) are further simplified:

$$
\begin{array}{rlc}
{\left[t_{0}\right] \Phi_{\ell, k, i}=\left(\begin{array}{ccc}
2 \mu+\lambda\left(\alpha_{k}+\ell+i+1\right) & (\lambda+2 \mu) \partial_{\varphi} & 0 \\
\mu \partial_{\varphi} & \mu\left(\alpha_{k}+\ell+i-1\right) & 0 \\
0 & 0 & \mu \partial_{\varphi}
\end{array}\right) \phi_{\ell, k, i},} \\
{\left[t_{01}\right] \Phi_{\ell, k, i}=\left(\begin{array}{ccc}
-\lambda & 0 & 0 \\
0 & 0 & 0 \\
0 & 0 & 0
\end{array}\right) \phi_{\ell, k, i},} & {\left[t_{1}\right] \Phi_{\ell, k, i}=\left(\begin{array}{ccc}
0 & 0 & \lambda \\
0 & 0 & 0 \\
0 & \mu & 0
\end{array}\right) \phi_{\ell, k, i}}
\end{array}
$$


The solution to the system (86) with traction free BCs (87) for a penny shaped crack $(\omega=2 \pi)$ is:

$$
\begin{aligned}
& \left\{\begin{array}{l}
u_{\rho} \\
u_{\varphi} \\
u_{\theta}
\end{array}\right\}=A_{1}\left(\begin{array}{c}
\cos \varphi \\
-\sin \varphi \\
0
\end{array}\right)\left[1-\left(\frac{\rho}{R}\right) \cos \varphi+\left(\frac{\rho}{R}\right)^{2} \cos ^{2} \varphi+\cdots\right]+A_{2}\left(\begin{array}{c}
\sin \varphi \\
\cos \varphi \\
0
\end{array}\right)+A_{3}\left(\begin{array}{l}
0 \\
0 \\
1
\end{array}\right)\left[1+\left(\frac{\rho}{R}\right) \cos \varphi\right] \\
& +A_{4} \rho^{\frac{1}{2}}\left[\left(\begin{array}{c}
-\frac{\lambda+5 \mu}{\lambda+\mu} \cos \frac{\varphi}{2}+\cos \frac{3 \varphi}{2} \\
\frac{3 \lambda+7 \mu}{\lambda+\mu} \sin \frac{\varphi}{2}-\sin \frac{3 \varphi}{2} \\
0
\end{array}\right)+\left(\frac{\rho}{R}\right)\left(\begin{array}{c}
\frac{3 \lambda^{2}+22 \lambda \mu-13 \mu^{2}}{12(\lambda+\mu)^{2}} \cos \frac{\varphi}{2}+\left(\frac{3}{4}-\frac{\lambda}{\lambda+\mu}\right) \cos \frac{3 \varphi}{2} \\
\frac{9 \lambda^{2}-14 \lambda \mu-55 \mu^{2}}{12(\lambda+\mu)^{2}} \sin \frac{\varphi}{2}+\left(-\frac{5}{4}+\frac{\lambda}{\lambda+\mu}\right) \sin \frac{3 \varphi}{2} \\
0
\end{array}\right)\right. \\
& \left.+\left(\frac{\rho}{R}\right)^{2}\left(\begin{array}{c}
-\frac{3 \lambda^{2}+134 \lambda \mu+67 \mu^{2}}{96(\lambda+\mu)^{2}} \cos \frac{\varphi}{2}-\frac{45 \lambda^{3}+255 \lambda^{2} \mu+199 \lambda \mu^{2}+53 \mu^{3}}{180(\lambda+\mu)^{3}} \cos \frac{3 \varphi}{2}+\frac{3(3 \lambda-\mu)}{32(\lambda+\mu)} \cos \frac{5 \varphi}{2} \\
\frac{69 \lambda^{2}+170 \lambda \mu+37 \mu^{2}}{96(\lambda+\mu)^{2}} \sin \frac{\varphi}{2}+\frac{-15 \lambda^{3}+155 \lambda^{2} \mu+371 \lambda \mu^{2}+137 \mu^{3}}{180(\lambda+\mu)^{3}} \sin \frac{3 \varphi}{2}-\frac{3(\lambda-3 \mu)}{32(\lambda+\mu)} \sin \frac{5 \varphi}{2} \\
0
\end{array}\right)+\cdots\right] \\
& +A_{5} \rho^{\frac{1}{2}}\left[\left(\begin{array}{c}
-\frac{\lambda+5 \mu}{3(\lambda+\mu)} \sin \frac{\varphi}{2}+\sin \frac{3 \varphi}{2} \\
-\frac{3 \lambda+7 \mu}{3(\lambda+\mu)} \cos \frac{\varphi}{2}+\cos \frac{3 \varphi}{2} \\
0
\end{array}\right)+\left(\frac{\rho}{R}\right)\left(\begin{array}{c}
-\frac{123 \lambda^{2}+262 \lambda \mu+107 \mu^{2}}{180(\lambda+\mu)^{2}} \sin \frac{\varphi}{2}-\frac{\lambda-3 \mu}{12(\lambda+\mu)} \sin \frac{3 \varphi}{2} \\
-\frac{15 \lambda^{2}+62 \lambda \mu+79 \mu^{2}}{180(\lambda+\mu)^{2}} \cos \frac{\varphi}{2}+\frac{(\lambda+5 \mu)}{12(\lambda+\mu)} \cos \frac{3 \varphi}{2} \\
0
\end{array}\right)\right. \\
& +\left(\frac{\rho}{R}\right)^{2} \frac{1}{1440}\left(\begin{array}{c}
-\frac{483 \lambda^{2}+1350 \lambda \mu+803 \mu^{2}}{(\lambda+\mu)^{2}} \sin \frac{\varphi}{2}+16 \frac{2625 \lambda^{3}+8779 \lambda^{2} \mu+9251 \lambda \mu^{2}+3161 \mu^{3}}{35(\lambda+\mu)^{3}} \sin \frac{3 \varphi}{2}+45 \frac{3 \lambda-\mu}{(\lambda+\mu)} \sin \frac{5 \varphi}{2} \\
-16 \frac{357 \lambda^{2}+1002 \lambda \mu+581 \mu^{2}}{(\lambda+\mu)^{2}} \cos \frac{\varphi}{2}+\frac{1365 \lambda^{3}+5271 \lambda^{2} \mu+6719 \lambda \mu^{2}+2749 \mu^{3}}{35(\lambda+\mu)^{3}} \cos \frac{3 \varphi}{2}+45 \frac{\lambda-3 \mu}{(\lambda+\mu)} \cos \frac{5 \varphi}{2} \\
0
\end{array}\right) \\
& +\cdots] \\
& +A_{6} \rho^{\frac{1}{2}}\left[\left(\begin{array}{c}
0 \\
0 \\
\sin \frac{\varphi}{2}
\end{array}\right)+\left(\frac{\rho}{R}\right)\left(\begin{array}{c}
0 \\
0 \\
\frac{1}{4} \sin \frac{\varphi}{2}
\end{array}\right)+\left(\frac{\rho}{R}\right)^{2}\left(\begin{array}{c}
0 \\
0 \\
\frac{1}{4} \sin \frac{\varphi}{2}-\frac{3}{32} \sin \frac{3 \varphi}{2}
\end{array}\right)+\cdots\right] \\
& +\cdots
\end{aligned}
$$

One may notice that the solutions of $\phi_{\rho}$ and $\phi_{\varphi}$ are decoupled from the solution of $\phi_{\theta}$. The eigenfunctions $\phi_{\theta}$ are not identical to the ones obtained by the Laplace equation - if one observes the last line in (90) in comparison with (19), then the coefficient of the term $\rho^{1 / 2}\left(\frac{\rho}{R}\right)^{2} \sin \frac{\varphi}{2}$ is $\frac{1}{4}$ instead of $\frac{1}{12}$ in (19). More differences would appear if inspecting terms of higher orders of $\frac{\rho}{R}$.

Remark 8. The second and third terms corresponding to zero eigen-values are associated with rigid body motions: The term $A_{2}(\sin \varphi, \cos \varphi, 0)^{\top}$ expressed in Cartesian components becomes $\left(u_{1}, u_{2}, u_{3}\right)_{2}^{\top}=$ $A_{2}(0,0,1)^{\top}$, which is clearly a translation along $z_{3}$. Likewise, the factor term of $A_{3}$ becomes $\left(u_{1}, u_{2}, u_{3}\right)_{3}^{\top}=A_{3} \frac{r}{R}(-\sin \theta, \cos \theta, 0)^{\top}$, which is proportional to the rotation $\left(-x_{2}, x_{1}, 0\right)^{\top}$ around the axis of symmetry. Note finally that for $\rho / R \ll 1$

$$
\frac{1}{1+\frac{\rho}{R} \cos \varphi}=\left[1-\frac{\rho}{R} \cos \varphi+\left(\frac{\rho}{R} \cos \varphi\right)^{2}-\left(\frac{\rho}{R} \cos \varphi\right)^{3}+\cdots\right]
$$

therefore the factor of $A_{1}$ is not linear in Cartesian coordinates: $\left(u_{1}, u_{2}, u_{3}\right)_{1}^{\top}=A_{1} R\left(\frac{x_{1}}{r^{2}}, \frac{x_{2}}{r^{2}}, 0\right)^{\top}$. Details on the derivation of the series solution associated with $A_{1}, A_{2}, A_{3}$ are provided in Appendix A.

Remark 9. The solution for the circular singular crack with traction free boundary condition was presented also by Leung and $\mathrm{Su}$ in [7], and is almost identical to (90). One difference is the factor $1 / 1440$ in the term associated with $A_{2} \rho^{\frac{1}{2}}\left(\frac{\rho}{R}\right)^{2}$ whereas in [7] the factor is $1 / 1400$. The solution herein is the correct one because it satisfies (86), whereas Leung and Su's solution does not. The second difference is in the shadow terms associated with $\alpha_{1}=0$ and $\alpha_{3}=0$ for $A_{1}$ and $A_{3}$ which are missing in Leung and Su's solution. 
Defining:

$$
K_{1} \stackrel{\text { def }}{=} A_{1} \mu, \quad\left(\frac{-1}{4}\right) \frac{K_{I}}{\sqrt{2 \pi}} \stackrel{\text { def }}{=} A_{4} \mu, \quad\left(\frac{3}{4}\right) \frac{K_{I I}}{\sqrt{2 \pi}} \stackrel{\text { def }}{=} A_{5} \mu, \quad 2 \frac{K_{I I I}}{\sqrt{2 \pi}} \stackrel{\text { def }}{=} A_{6} \mu
$$

with $K_{I}, K_{I I}, K_{I I I}$ the renown stress intensity factors in fracture mechanics, and using (51)-(55), the stress tensor associated with an axisymmetric traction free problem is:

$$
\begin{aligned}
& \left\{\begin{array}{c}
\sigma_{\rho \rho} \\
\sigma_{\theta \theta} \\
\sigma_{\varphi \varphi} \\
\sigma_{\rho \theta} \\
\sigma_{\rho \varphi} \\
\sigma_{\theta \varphi}
\end{array}\right\}=K_{1}\left(\begin{array}{c}
-1-\cos 2 \varphi \\
2 \\
-1+\cos 2 \varphi \\
0 \\
\sin 2 \varphi \\
0
\end{array}\right)\left(\frac{\rho}{R}\right)\left[1-2 \cos \varphi\left(\frac{\rho}{R}\right)+3 \cos ^{2} \varphi\left(\frac{\rho}{R}\right)^{2}-4 \cos ^{3} \varphi\left(\frac{\rho}{R}\right)^{3}+\cdots\right] \\
& +\frac{-1}{4} \frac{K_{I}}{\sqrt{2 \pi \rho}}\left[\left(\begin{array}{c}
-5 \cos \frac{\varphi}{2}+\cos \frac{3 \varphi}{2} \\
-\frac{4 \lambda}{\lambda+\mu} \cos \frac{\varphi}{2} \\
-3 \cos \frac{\varphi}{2}-\cos \frac{3 \varphi}{2} \\
0 \\
-\sin \frac{\varphi}{2}-\sin \frac{3 \varphi}{2} \\
0
\end{array}\right)+\left(\frac{\rho}{R}\right)\left(\begin{array}{c}
-\frac{5 \lambda+13 \mu}{4(\lambda+\mu)} \cos \frac{\varphi}{2}+\frac{\lambda+9 \mu}{4(\lambda+\mu)} \cos \frac{3 \varphi}{2} \\
-\frac{2(2 \lambda+\mu)(\lambda+5 \mu)}{(\lambda+\mu)^{2}} \cos \frac{\varphi}{2}+\frac{3 \lambda+2 \mu}{\lambda+\mu} \cos \frac{3 \varphi}{2} \\
-\frac{3(\lambda+9 \mu)}{4(\lambda+\mu)} \cos \frac{\varphi}{2}-\frac{\lambda+9 \mu}{4(\lambda+\mu)} \cos \frac{3 \varphi}{2} \\
0 \\
\frac{\lambda-7 \mu}{4(\lambda+\mu)} \sin \frac{\varphi}{2}+\frac{\lambda-7 \mu}{4(\lambda+\mu)} \sin \frac{3 \varphi}{2} \\
0
\end{array}\right)+\cdots\right] \\
& +\frac{3}{4} \frac{K_{I I}}{\sqrt{2 \pi \rho}}\left[\left(\begin{array}{c}
-\frac{5}{3} \sin \frac{\varphi}{2}+\sin \frac{3 \varphi}{2} \\
-\frac{4 \lambda}{3(\lambda+\mu)} \sin \frac{\varphi}{2} \\
-\sin \frac{\varphi}{2}-\sin \frac{3 \varphi}{2} \\
0 \\
\frac{1}{3}\left(\begin{array}{c}
\left.\cos \frac{\varphi}{2}+3 \cos \frac{3 \varphi}{2}\right) \\
0
\end{array}\right)+\left(\frac{\rho}{R}\right)\left(\begin{array}{c}
-\frac{51 \lambda+107 \mu}{60(\lambda+\mu)} \sin \frac{\varphi}{2}+\frac{\lambda+9 \mu}{12(\lambda+\mu)} \sin \frac{3 \varphi}{2} \\
\frac{2\left(34 \lambda^{2}+83 \lambda \mu+45 \mu^{2}\right)}{15(\lambda+\mu)^{2}} \sin \frac{\varphi}{2}+\frac{3 \lambda+2 \mu}{3(\lambda+\mu)} \sin \frac{3 \varphi}{2} \\
-\frac{\lambda+9 \mu}{12(\lambda+\mu)} \sin \frac{\varphi}{2}-\frac{\lambda+9 \mu}{12(\lambda+\mu)} \sin \frac{3 \varphi}{2} \\
0 \\
-\frac{23 \lambda+31 \mu}{60(\lambda+\mu)} \cos \frac{\varphi}{2}+\frac{-\lambda+7 \mu}{12(\lambda+\mu)} \cos \frac{3 \varphi}{2} \\
0
\end{array}\right)+\cdots
\end{array}\right]\right. \\
& +2 \frac{K_{I I I}}{\sqrt{2 \pi \rho}}\left[\left(\begin{array}{c}
0 \\
0 \\
0 \\
\frac{1}{2} \sin \frac{\varphi}{2} \\
0 \\
\frac{1}{2} \cos \frac{\varphi}{2}
\end{array}\right)+\left(\frac{\rho}{R}\right)\left(\begin{array}{c}
0 \\
0 \\
0 \\
\frac{7}{8} \sin \frac{\varphi}{2}-\frac{1}{2} \sin \frac{3 \varphi}{2} \\
0 \\
\frac{5}{8} \cos \frac{\varphi}{2}-\frac{1}{2} \cos \frac{3 \varphi}{2}
\end{array}\right)+\cdots\right]
\end{aligned}
$$

Remark 10. For $R \rightarrow \infty$ the stresses state should tend to a plane-strain state. Indeed, by computing $\nu\left(\sigma_{\rho \rho}+\sigma_{\varphi \varphi}\right)$ from (91), one obtains $\nu\left[2 \frac{K_{I}}{\sqrt{2 \pi \rho}} \cos \frac{\varphi}{2}-2 \frac{K_{I I}}{\sqrt{2 \pi \rho}} \sin \frac{\varphi}{2}\right]$ that equals $\sigma_{\theta \theta}$. This is exactly the connection: $\sigma_{33}=\nu\left(\sigma_{11}+\sigma_{22}\right)$ according to a plane-strain situation.

Remark 11. The primal eigen-stresses $\sigma_{\rho \rho}$ and $\sigma_{\varphi \varphi}$ do not depend on the material properties for traction free boundary conditions on crack faces. However, their shadows do depend on the material properties.

3.2.2. A specific example problem - penny-shaped crack with axisymmetric loading and clamped BCs. For the clamped BCs (Dirichlet BCs) and a penny shaped crack $(\omega=2 \pi)$ the series expansion of the 
solution is:

$$
\begin{aligned}
& \left\{\begin{array}{l}
u_{\rho} \\
u_{\varphi} \\
u_{\theta}
\end{array}\right\}=A_{1} \rho^{\frac{1}{2}}\left[\left(\begin{array}{c}
\frac{\lambda+5 \mu}{3 \lambda+7 \mu} \cos \frac{\varphi}{2}+\cos \frac{3 \varphi}{2} \\
-\sin \frac{\varphi}{2}-\sin \frac{3 \varphi}{2} \\
0
\end{array}\right)+\left(\frac{\rho}{R}\right)\left(\begin{array}{c}
-\frac{41 \lambda^{2}+154 \lambda \mu+129 \mu^{2}}{4(3 \lambda+7 \mu)(5 \lambda+9 \mu)} \cos \frac{\varphi}{2}+\frac{\lambda-3 \mu}{4(3 \lambda+7 \mu)} \cos \frac{3 \varphi}{2} \\
\frac{\lambda+5 \mu}{4(3 \lambda+7 \mu)} \sin \frac{\varphi}{2}+\frac{\lambda+5 \mu}{4(3 \lambda+7 \mu)} \sin \frac{3 \varphi}{2} \\
0
\end{array}\right)\right. \\
& \left.+\left(\frac{\rho}{R}\right)^{2}\left(\begin{array}{c}
\frac{(7 \lambda+11 \mu)(23 \lambda+67 \mu)}{32(3 \lambda+7 \mu)(5 \lambda+9 \mu)} \cos \frac{\varphi}{2}+\frac{25 \lambda^{2}+98 \lambda \mu+89 \mu^{2}}{4(3 \lambda+7 \mu)(5 \lambda+9 \mu)} \cos \frac{3 \varphi}{2}+\frac{3(-3 \lambda+\mu)}{32(3 \lambda+7 \mu)} \cos \frac{5 \varphi}{2} \\
-\frac{(7 \lambda+11 \mu)(17 \lambda+37 \mu)}{32(3 \lambda+7 \mu)(5 \lambda+9 \mu)} \sin \frac{\varphi}{2}-\frac{13 \lambda^{2}+58 \lambda \mu+61 \mu^{2}}{4(3 \lambda+7 \mu)(5 \lambda+9 \mu)} \sin \frac{3 \varphi}{2}+\frac{3(\lambda-3 \mu)}{32(3 \lambda+7 \mu)} \sin \frac{5 \varphi}{2} \\
0
\end{array}\right)+\cdots\right] \\
& +A_{2} \rho^{\frac{1}{2}}\left[\left(\begin{array}{c}
\sin \frac{\varphi}{2}+\sin \frac{3 \varphi}{2} \\
\frac{3 \lambda+7 \mu}{\lambda+5 \mu} \cos \frac{\varphi}{2}+\cos \frac{3 \varphi}{2} \\
0
\end{array}\right)+\left(\frac{\rho}{R}\right)\left(\begin{array}{c}
\frac{\lambda-3 \mu}{4(\lambda+5 \mu)} \sin \frac{\varphi}{2}+\frac{\lambda-3 \mu}{4(\lambda+5 \mu)} \sin \frac{3 \varphi}{2} \\
-\frac{3 \lambda+7 \mu}{4(\lambda+5 \mu)} \cos \frac{\varphi}{2}-\frac{1}{4} \cos \frac{3 \varphi}{2} \\
0
\end{array}\right)\right. \\
& \left.+\left(\frac{\rho}{R}\right)^{2}\left(\begin{array}{c}
\frac{3 \lambda-\mu}{96(\lambda+5 \mu)} \sin \frac{\varphi}{2}+\frac{-3 \lambda+\mu}{12(\lambda+5 \mu)} \sin \frac{3 \varphi}{2}+\frac{3(-3 \lambda+\mu)}{32(\lambda+5 \mu)} \sin \frac{5 \varphi}{2} \\
\frac{69 \lambda+137 \mu}{96(\lambda+5 \mu)} \cos \frac{\varphi}{2}+\frac{1}{12} \cos \frac{3 \varphi}{2}+-\frac{3(\lambda-3 \mu)}{32(\lambda+5 \mu)} \cos \frac{5 \varphi}{2} \\
0
\end{array}\right)+\cdots\right] \\
& +A_{3} \rho^{\frac{1}{2}}\left[\left(\begin{array}{c}
0 \\
0 \\
\cos \frac{\varphi}{2}
\end{array}\right)+\left(\frac{\rho}{R}\right)\left(\begin{array}{c}
0 \\
0 \\
-\frac{1}{4} \cos \frac{\varphi}{2}
\end{array}\right)+\left(\frac{\rho}{R}\right)^{2}\left(\begin{array}{c}
0 \\
0 \\
\frac{1}{4} \cos \frac{\varphi}{2}+\frac{3}{32} \cos \frac{3 \varphi}{2}
\end{array}\right)+\cdots\right]
\end{aligned}
$$

Here we define:

$$
A_{1} \stackrel{\text { def }}{=} \frac{3 \lambda+7 \mu}{-4 \mu} \frac{K_{I}}{\sqrt{2 \pi}}, \quad A_{2} \stackrel{\text { def }}{=} \frac{\lambda+5 \mu}{4 \mu} \frac{K_{I I}}{\sqrt{2 \pi}}, \quad A_{3} \stackrel{\text { def }}{=} 2 \frac{K_{I I I}}{\sqrt{2 \pi}}
$$

then the corresponding stress tensor is:

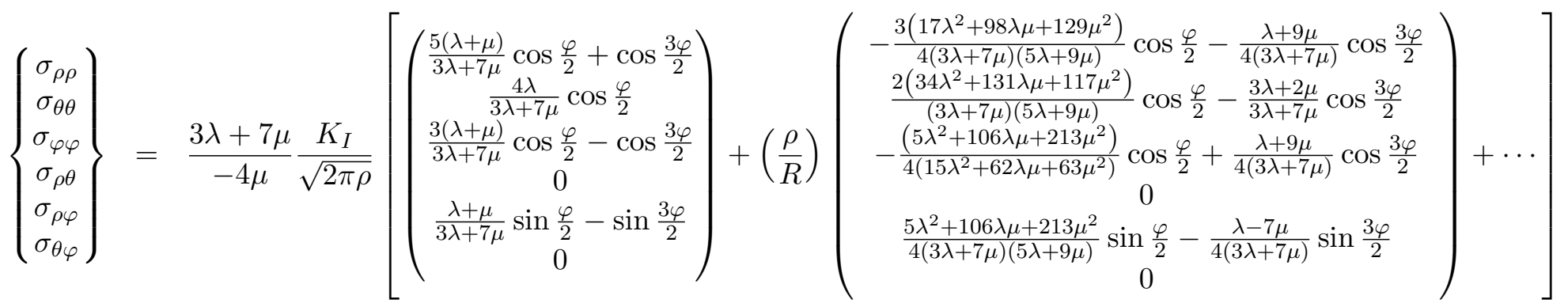

$$
\begin{aligned}
& +\frac{\lambda+5 \mu}{4 \mu} \frac{K_{I I}}{\sqrt{2 \pi \rho}}\left[\left(\begin{array}{c}
\frac{5(\lambda+\mu)}{\lambda+5 \mu} \sin \frac{\varphi}{2}+\sin \frac{3 \varphi}{2} \\
\frac{4 \lambda}{\lambda+5 \mu} \sin \frac{\varphi}{2} \\
\frac{3(\lambda+\mu)}{\lambda+5 \mu} \sin \frac{\varphi}{2}-\sin \frac{3 \varphi}{2} \\
0 \\
-\frac{\lambda+\mu}{\lambda+5 \mu} \cos \frac{\varphi}{2}+\cos \frac{3 \varphi}{2} \\
0
\end{array}\right)+\left(\frac{\rho}{R}\right)\left(\begin{array}{c}
-\frac{5 \lambda+9 \mu}{4(\lambda+5 \mu)} \sin \frac{\varphi}{2}-\frac{\lambda+9 \mu}{4(\lambda+5 \mu)} \sin \frac{3 \varphi}{2} \\
-\frac{2(2 \lambda+\mu)}{\lambda+5 \mu} \sin \frac{\varphi}{2}-\frac{3 \lambda+2 \mu}{\lambda+5 \mu} \sin \frac{3 \varphi}{2} \\
\frac{-3 \lambda+\mu}{4(\lambda+5 \mu)} \sin \frac{\varphi}{2}+\frac{\lambda+9 \mu}{4(\lambda+5 \mu)} \sin \frac{3 \varphi}{2} \\
0 \\
-\frac{\lambda+5 \mu}{4(\lambda+5 \mu)} \cos \frac{\varphi}{2}+\frac{\lambda-7 \mu}{4(\lambda+5 \mu)} \cos \frac{3 \varphi}{2} \\
0
\end{array}\right]+\cdots\right] \\
& +2 \frac{K_{I I I}}{\sqrt{2 \pi \rho}}\left[\left(\begin{array}{c}
0 \\
0 \\
0 \\
\frac{1}{2} \cos \frac{\varphi}{2} \\
0 \\
-\frac{1}{2} \sin \frac{\varphi}{2}
\end{array}\right)+\left(\frac{\rho}{R}\right)\left(\begin{array}{c}
0 \\
0 \\
0 \\
-\frac{7}{8} \cos \frac{\varphi}{2}-\frac{1}{2} \cos \frac{3 \varphi}{2} \\
0 \\
\frac{5}{8} \sin \frac{\varphi}{2}+\frac{1}{2} \sin \frac{3 \varphi}{2}
\end{array}\right)+\cdots\right]
\end{aligned}
$$


3.3. Non axi-symmetric case. The set of equations corresponding to $\ell=0$ is exactly the axisymmertric set of equations presented in section 3.2. Herein we present the entire solution including the terms for $\ell \geq 1$ for a non axi-symmetric case.

3.3.1. A specific example problem - penny-shaped crack with non-axisymmetric loading and traction free BCs. For the traction free BCs (82) and a penny shaped crack $(\omega=2 \pi)$ we solved the system (70), obtaining:

$$
\begin{aligned}
& \left\{\begin{array}{l}
u_{\rho} \\
u_{\varphi} \\
u_{\theta}
\end{array}\right\}=A_{1}(\theta)\left(\begin{array}{c}
\cos \varphi \\
-\sin \varphi \\
0
\end{array}\right)\left[1-\left(\frac{\rho}{R}\right) \cos \varphi+\left(\frac{\rho}{R}\right)^{2} \cos ^{2} \varphi+\cdots\right]+A_{1}^{\prime}(\theta)\left(\frac{\rho}{R}\right)\left[\left(\frac{\rho}{R}\right)\left(\begin{array}{c}
0 \\
0 \\
-\cos ^{2} \varphi
\end{array}\right)+\cdots\right] \\
& +A_{1}^{\prime \prime}(\theta)\left(\frac{\rho}{R}\right)^{2}\left(\begin{array}{c}
-\frac{\lambda+2 \mu}{12(\lambda+\mu)} \cos \varphi \\
\frac{3 \lambda+2 \mu}{12(\lambda+\mu)} \sin \varphi \\
0
\end{array}\right)+\cdots \\
& +A_{2}(\theta)\left(\begin{array}{c}
\sin \varphi \\
\cos \varphi \\
0
\end{array}\right)+A_{2}^{\prime}(\theta)\left(\frac{\rho}{R}\right)\left[\left(\begin{array}{c}
0 \\
0 \\
-\sin \varphi
\end{array}\right)+\left(\frac{\rho}{R}\right)\left(\begin{array}{c}
0 \\
0 \\
\frac{1}{2} \sin 2 \varphi
\end{array}\right)+\cdots\right] \\
& +A_{2}^{\prime \prime}(\theta)\left(\frac{\rho}{R}\right)^{2}\left(\begin{array}{c}
\frac{\lambda}{4(\lambda+\mu)} \sin \varphi \\
-\frac{\lambda}{4(\lambda+\mu)} \cos \varphi \\
0
\end{array}\right)+\cdots \\
& +A_{3}(\theta)\left(\begin{array}{l}
0 \\
0 \\
1
\end{array}\right)\left[1+\left(\frac{\rho}{R}\right) \cos \varphi\right]+A_{3}^{\prime}(\theta)\left(\frac{\rho}{R}\right)\left[\left(\begin{array}{c}
-\frac{\lambda}{2(\lambda+\mu)} \\
0 \\
0
\end{array}\right)+\left(\frac{\rho}{R}\right)\left(\begin{array}{c}
\frac{9 \lambda^{2}+16 \lambda \mu+8 \mu^{2}}{24(\lambda+\mu)^{2}} \cos \varphi \\
-\frac{15 \lambda^{2}+24 \lambda \mu+8 \mu^{2}}{24(\lambda+\mu)^{2}} \sin \varphi \\
0
\end{array}\right) \cdots\right] \\
& +A_{3}^{\prime \prime}(\theta)\left(\frac{\rho}{R}\right)^{2}\left(\begin{array}{c}
0 \\
0 \\
-\frac{1}{2}
\end{array}\right)+\cdots \\
& +A_{4}(\theta) \rho^{\frac{1}{2}}\left[\left(\begin{array}{c}
-\frac{\lambda+5 \mu}{\lambda+\mu} \cos \frac{\varphi}{2}+\cos \frac{3 \varphi}{2} \\
\frac{3 \lambda+7 \mu}{\lambda+\mu} \sin \frac{\varphi}{2}-\sin \frac{3 \varphi}{2} \\
0
\end{array}\right)+\left(\frac{\rho}{R}\right)\left(\begin{array}{c}
\frac{3 \lambda^{2}+22 \lambda \mu-13 \mu^{2}}{12(\lambda+\mu)^{2}} \cos \frac{\varphi}{2}+\left(\frac{3}{4}-\frac{\lambda}{\lambda+\mu}\right) \cos \frac{3 \varphi}{2} \\
\frac{9 \lambda^{2}-14 \lambda \mu-55 \mu^{2}}{12(\lambda+\mu)^{2}} \sin \frac{\varphi}{2}+\left(-\frac{5}{4}+\frac{\lambda}{\lambda+\mu}\right) \sin \frac{3 \varphi}{2} \\
0
\end{array}\right)\right.
\end{aligned}
$$

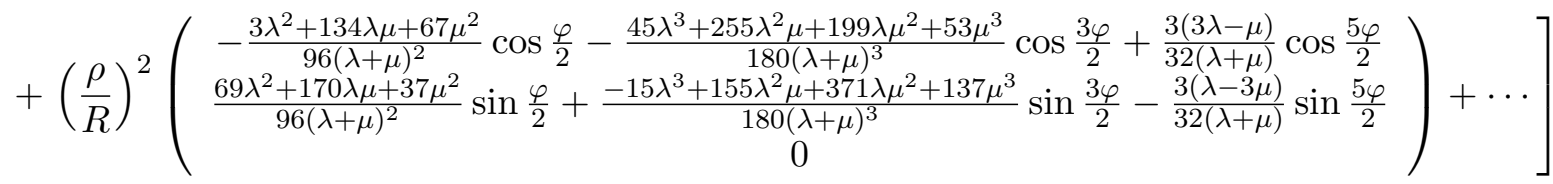

$$
\begin{aligned}
& +A_{4}^{\prime}(\theta) \rho^{\frac{1}{2}}\left(\frac{\rho}{R}\right)\left[\left(\begin{array}{c}
0 \\
0 \\
2 \cos \frac{\varphi}{2}-\frac{2(3 \lambda+7 \mu)}{3(\lambda+\mu)} \cos \frac{3 \varphi}{2}
\end{array}\right)\right. \\
& \left.+\left(\frac{\rho}{R}\right)\left(\begin{array}{c}
0 \\
0 \\
\frac{3 \lambda+23 \mu}{6(\lambda+\mu)} \cos \frac{\varphi}{2}-\frac{3}{2} \cos \frac{3 \varphi}{2}+\frac{15 \lambda^{2}+34 \lambda \mu+3 \mu^{2}}{15(\lambda+\mu)^{2}} \cos \frac{5 \varphi}{2}
\end{array}\right)+\cdots\right] \\
& +A_{4}^{\prime \prime}(\theta) \rho^{\frac{1}{2}}\left(\frac{\rho}{R}\right)^{2}\left(\begin{array}{c}
-\frac{3 \lambda-\mu}{6(\lambda+\mu)} \cos \frac{\varphi}{2}+\frac{45 \lambda^{2}+138 \lambda \mu+61 \mu^{2}}{90(\lambda+\mu)^{2}} \cos \frac{3 \varphi}{2} \\
-\frac{3 \lambda+7 \mu}{6(\lambda+\mu)} \sin \frac{\varphi}{2}+\frac{15 \lambda^{2}-2 \lambda \mu-49 \mu^{2}}{90(\lambda+\mu)^{2}} \sin \frac{3 \varphi}{2} \\
0
\end{array}\right) \quad+\cdots / \cdots
\end{aligned}
$$




$$
\begin{aligned}
& \cdots+A_{5}(\theta) \rho^{\frac{1}{2}}\left[\left(\begin{array}{c}
-\frac{\lambda+5 \mu}{3(\lambda+\mu)} \sin \frac{\varphi}{2}+\sin \frac{3 \varphi}{2} \\
-\frac{3 \lambda+7 \mu}{3(\lambda+\mu)} \cos \frac{\varphi}{2}+\cos \frac{3 \varphi}{2} \\
0
\end{array}\right)+\left(\frac{\rho}{R}\right)\left(\begin{array}{c}
-\frac{123 \lambda^{2}+262 \lambda \mu+107 \mu^{2}}{180(\lambda+\mu)^{2}} \sin \frac{\varphi}{2}-\frac{\lambda-3 \mu}{12(\lambda+\mu)} \sin \frac{3 \varphi}{2} \\
-\frac{15 \lambda^{2}+62 \lambda \mu+79 \mu^{2}}{180(\lambda+\mu)^{2}} \cos \frac{\varphi}{2}+\frac{(\lambda+5 \mu)}{12(\lambda+\mu)} \cos \frac{3 \varphi}{2} \\
0
\end{array}\right)\right. \\
& \left.+\left(\frac{\rho}{R}\right)^{2}\left(\begin{array}{c}
-\frac{483 \lambda^{2}+1350 \lambda \mu+803 \mu^{2}}{1440(\lambda+\mu)^{2}} \sin \frac{\varphi}{2}+\frac{2625 \lambda^{3}+8779 \lambda^{2} \mu+9251 \lambda \mu^{2}+3161 \mu^{3}}{6300(\lambda+\mu)^{3}} \sin \frac{3 \varphi}{2}+\frac{3 \lambda-\mu}{32(\lambda+\mu)} \sin \frac{5 \varphi}{2} \\
-\frac{357 \lambda^{2}+1002 \lambda \mu+581 \mu^{2}}{1440(\lambda+\mu)^{2}} \cos \frac{\varphi}{2}+\frac{1365 \lambda^{3}+5271 \lambda^{2} \mu+6719 \lambda \mu^{2}+2749 \mu^{3}}{6300(\lambda+\mu)^{3}} \cos \frac{3 \varphi}{2}+\frac{\lambda-3 \mu}{32(\lambda+\mu)} \cos \frac{5 \varphi}{2} \\
0
\end{array}\right)+\cdots\right] \\
& +A_{5}^{\prime}(\theta) \rho^{\frac{1}{2}}\left(\frac{\rho}{R}\right)\left[\left(\begin{array}{c}
0 \\
0 \\
\frac{2}{3} \sin \frac{\varphi}{2}
\end{array}\right)+\left(\frac{\rho}{R}\right)\left(\begin{array}{c}
0 \\
0 \\
-\frac{8(\lambda+2 \mu)}{15(\lambda+\mu)} \sin \frac{\varphi}{2}-\frac{1}{2} \sin \frac{3 \varphi}{2}
\end{array}\right)+\cdots\right] \\
& +A_{5}^{\prime \prime}(\theta) \rho^{\frac{1}{2}}\left(\frac{\rho}{R}\right)^{2}\left(\begin{array}{c}
\left(\frac{1}{18}-\frac{2 \lambda}{9(\lambda+\mu)}\right) \sin \frac{\varphi}{2}-\frac{45 \lambda^{2}+90 \lambda \mu+77 \mu^{2}}{630(\lambda+\mu)^{2}} \sin \frac{3 \varphi}{2} \\
\left(\frac{7}{18}-\frac{2 \lambda}{9(\lambda+\mu)}\right) \cos \frac{\varphi}{2}+\frac{-105 \lambda^{2}-130 \lambda \mu+7 \mu^{2}}{630(\lambda+\mu)^{2}} \cos \frac{3 \varphi}{2} \\
0
\end{array}\right)+\cdots \\
& +A_{6}(\theta) \rho^{\frac{1}{2}}\left[\left(\begin{array}{c}
0 \\
0 \\
\sin \frac{\varphi}{2}
\end{array}\right)+\left(\frac{\rho}{R}\right)\left(\begin{array}{c}
0 \\
0 \\
\frac{1}{4} \sin \frac{\varphi}{2}
\end{array}\right)+\left(\frac{\rho}{R}\right)^{2}\left(\begin{array}{c}
0 \\
0 \\
\frac{1}{4} \sin \frac{\varphi}{2}-\frac{3}{32} \sin \frac{3 \varphi}{2}
\end{array}\right)+\cdots\right] \\
& +A_{6}^{\prime}(\theta) \rho^{\frac{1}{2}}\left(\frac{\rho}{R}\right)\left[\left(\begin{array}{c}
-\frac{2(3 \lambda+\mu)}{15(\lambda+\mu)} \sin \frac{\varphi}{2} \\
-\frac{4 \mu}{15(\lambda+\mu)} \cos \frac{\varphi}{2} \\
0
\end{array}\right)+\left(\frac{\rho}{R}\right)\left(\begin{array}{c}
\left(-\frac{7}{30}-\frac{\lambda}{15(\lambda+\mu)}\right) \sin \frac{\varphi}{2}+\frac{2\left(75 \lambda^{2}+124 \lambda \mu+53 \mu^{2}\right)}{525(\lambda+\mu)^{2}} \sin \frac{3 \varphi}{2} \\
-\frac{3 \lambda+2 \mu}{15(\lambda+\mu)} \cos \frac{\varphi}{2}+\frac{105 \lambda^{2}+242 \lambda \mu+129 \mu^{2}}{525(\lambda+\mu)^{2}} \cos \frac{3 \varphi}{2} \\
0
\end{array}\right)+\cdots\right] \\
& +A_{6}^{\prime \prime}(\theta) \rho^{\frac{1}{2}}\left(\frac{\rho}{R}\right)^{2}\left(\begin{array}{c}
0 \\
0 \\
-\frac{3}{10} \sin \frac{\varphi}{2}
\end{array}\right)+\cdots \\
& +\ldots
\end{aligned}
$$


The stresses in this case with the definition of SIFs as in (90) are:

$$
\begin{aligned}
& \left\{\begin{array}{c}
\sigma_{\rho \rho} \\
\sigma_{\theta \theta} \\
\sigma_{\varphi \varphi} \\
\sigma_{\rho \theta} \\
\sigma_{\rho \varphi} \\
\sigma_{\theta \varphi}
\end{array}\right\}=K_{1}(\theta)\left(\begin{array}{c}
-1-\cos 2 \varphi \\
2 \\
-1+\cos 2 \varphi \\
0 \\
\sin 2 \varphi \\
0
\end{array}\right)\left(\frac{\rho}{R}\right)+K_{1}^{\prime}(\theta)\left(\frac{\rho}{R}\right)\left(\begin{array}{c}
0 \\
0 \\
0 \\
\cos \varphi \\
0 \\
-\sin \varphi
\end{array}\right)+\cdots \\
& +K_{2}^{\prime}(\theta)\left(\frac{\rho}{R}\right)^{2}\left(\begin{array}{c}
0 \\
0 \\
0 \\
\sin 2 \varphi \\
0 \\
-2 \sin ^{2} \varphi
\end{array}\right)+K_{3}^{\prime}(\theta)\left(\frac{\rho}{R}\right)\left(\begin{array}{c}
0 \\
\frac{3 \lambda+2 \mu}{\lambda+\mu} \\
0 \\
0 \\
0 \\
0
\end{array}\right)+\cdots \\
& +\left(\frac{-1}{4}\right) \frac{K_{I}(\theta)}{\sqrt{2 \pi \rho}}\left[\left(\begin{array}{c}
-5 \cos \frac{\varphi}{2}+\cos \frac{3 \varphi}{2} \\
-\frac{4 \lambda}{\lambda+\mu} \cos \frac{\varphi}{2} \\
-3 \cos \frac{\varphi}{2}-\cos \frac{3 \varphi}{2} \\
0 \\
-\sin \frac{\varphi}{2}-\sin \frac{3 \varphi}{2} \\
0
\end{array}\right)+\left(\frac{\rho}{R}\right)\left(\begin{array}{c}
-\frac{5 \lambda+13 \mu}{4(\lambda+\mu)} \cos \frac{\varphi}{2}+\frac{\lambda+9 \mu}{4(\lambda+\mu)} \cos \frac{3 \varphi}{2} \\
-\frac{2(2 \lambda+\mu)(\lambda+5 \mu)}{(\lambda+\mu)^{2}} \cos \frac{\varphi}{2}+\frac{3 \lambda+2 \mu}{\lambda+\mu} \cos \frac{3 \varphi}{2} \\
-\frac{3(\lambda+9 \mu)}{4(\lambda+\mu)} \cos \frac{\varphi}{2}-\frac{\lambda+9 \mu}{4(\lambda+\mu)} \cos \frac{3 \varphi}{2} \\
0 \\
\frac{\lambda-7 \mu}{4(\lambda+\mu)} \sin \frac{\varphi}{2}+\frac{\lambda-7 \mu}{4(\lambda+\mu)} \sin \frac{3 \varphi}{2} \\
0
\end{array}\right)+\cdots\right] \\
& +\left(\frac{-1}{4}\right) \frac{K_{I}^{\prime}(\theta)}{\sqrt{2 \pi \rho}}\left(\frac{\rho}{R}\right)\left(\begin{array}{c}
0 \\
0 \\
0 \\
\frac{2(\lambda-\mu)}{\lambda+\mu} \cos \frac{\varphi}{2}-\frac{2(\lambda+3 \mu)}{\lambda+\mu} \cos \frac{3 \varphi}{2} \\
0 \\
\frac{2(\lambda+3 \mu)}{\lambda+\mu} \sin \frac{\varphi}{2}+\frac{2(\lambda+3 \mu)}{\lambda+\mu} \sin \frac{3 \varphi}{2}
\end{array}\right)+\cdots \\
& +\frac{3}{4} \frac{K_{I I}(\theta)}{\sqrt{2 \pi \rho}}\left[\left(\begin{array}{c}
-\frac{5}{3} \sin \frac{\varphi}{2}+\sin \frac{3 \varphi}{2} \\
-\frac{4 \lambda}{3(\lambda+\mu)} \sin \frac{\varphi}{2} \\
-\sin \frac{\varphi}{2}-\sin \frac{3 \varphi}{2} \\
0 \\
\frac{1}{3}\left(\cos \frac{\varphi}{2}+3 \cos \frac{3 \varphi}{2}\right) \\
0
\end{array}\right)+\left(\frac{\rho}{R}\right)\left(\begin{array}{c}
-\frac{51 \lambda+107 \mu}{60(\lambda+\mu)} \sin \frac{\varphi}{2}+\frac{\lambda+9 \mu}{12(\lambda+\mu)} \sin \frac{3 \varphi}{2} \\
\frac{2\left(34 \lambda^{2}+83 \lambda \mu+45 \mu^{2}\right)}{15(\lambda+\mu)^{2}} \sin \frac{\varphi}{2}+\frac{3 \lambda+2 \mu}{3(\lambda+\mu)} \sin \frac{3 \varphi}{2} \\
-\frac{\lambda+9 \mu}{12(\lambda+\mu)} \sin \frac{\varphi}{2}-\frac{\lambda+9 \mu}{12(\lambda+\mu)} \sin \frac{3 \varphi}{2} \\
0 \\
-\frac{23 \lambda+31 \mu}{60(\lambda+\mu)} \cos \frac{\varphi}{2}+\frac{-\lambda+7 \mu}{12(\lambda+\mu)} \cos \frac{3 \varphi}{2} \\
0
\end{array}\right]+\cdots\right] \\
& +\frac{3}{4} \frac{K_{I I}^{\prime}(\theta)}{\sqrt{2 \pi \rho}}\left(\frac{\rho}{R}\right)\left(\begin{array}{c}
0 \\
0 \\
0 \\
\frac{2(\lambda-\mu)}{3(\lambda+\mu)} \sin \frac{\varphi}{2}+\sin \frac{3 \varphi}{2} \\
0 \\
-\frac{2(\lambda+3 \mu)}{3(\lambda+\mu)} \cos \frac{\varphi}{2}+\cos \frac{3 \varphi}{2}
\end{array}\right)+\cdots \\
& +2 \frac{K_{I I I}(\theta)}{\sqrt{2 \pi \rho}}\left[\left(\begin{array}{c}
0 \\
0 \\
0 \\
\frac{1}{2} \sin \frac{\varphi}{2} \\
0 \\
\frac{1}{2} \cos \frac{\varphi}{2}
\end{array}\right)+\left(\frac{\rho}{R}\right)\left(\begin{array}{c}
0 \\
0 \\
0 \\
\frac{7}{8} \sin \frac{\varphi}{2}-\frac{1}{2} \sin \frac{3 \varphi}{2} \\
0 \\
\frac{5}{8} \cos \frac{\varphi}{2}-\frac{1}{2} \cos \frac{3 \varphi}{2}
\end{array}\right)+\cdots\right]+2 \frac{K_{I I I}^{\prime}(\theta)}{\sqrt{2 \pi \rho}}\left(\frac{\rho}{R}\right)\left(\begin{array}{c}
-\frac{2}{5} \sin \frac{\varphi}{2} \\
\frac{2}{5} \frac{7 \lambda+5 \mu}{\lambda+\mu} \sin \frac{\varphi}{2} \\
0 \\
0 \\
-\frac{1}{5} \cos \frac{\varphi}{2} \\
0
\end{array}\right)+\cdots
\end{aligned}
$$

Remark 12. Comparing the terms associated with the first derivatives of the SIFs $\left(K_{I}^{\prime}(\theta), K_{I I}^{\prime}(\theta), K_{I I I}^{\prime}(\theta)\right.$ ) in [6] with (96), one notices that these are identical for $K_{I}^{\prime}(\theta)$ and $K_{I I I}^{\prime}(\theta)$. The term that multiplies $K_{I I}^{\prime}(\theta)$ in [6] appears in (96), but in our expression there are another two expressions proportional to $\cos \frac{3 \varphi}{2}$ and $\sin \frac{3 \varphi}{2}$ that are absent in [6]. 


\section{SUMMARY AND CONCLUSIONS}

We have formulated the set of recursive ODEs required for the representation of the asymptotic solution in the vicinity of a circular V-notch for the Laplace equation and the elasticity system in a 3-D setting. The asymptotic solution is constructed from 2-D eigen-pairs (a V-notch in a 2-D domain) complemented by two families of shadow functions. Both homogeneous Dirichlet (clamped) or homogeneous Neumann (traction free) boundary conditions were reformulated in terms of eigen-pairs and their shadows, and explicit representation of the series was computed for a penny shaped crack, a circumferential crack and an external crack as well as a $3 \pi / 2$ reentrant corner.

Having the eigen-pairs and their shadows available, one may construct bench-mark problems for the verification of various numerical schemes. In a future publication the quasidual-singular-function method [3] for the computation of the Edge-Flux/Stress-Intensity-Functions $A_{k}(\theta)$ will be extended to circular singular edges using the series expansion presented herein.

Acknowledgements: The authors gratefully acknowledge an anonymous referee for valuable and constructive comments, leading to improvements in the presentation and context. The first two authors gratefully acknowledge the support of this work by the Israel Science Foundation (grant No. 750/07). 


\section{APPENDiX A. DERIVATION OF THE SHADOW TERMS ASSOCIATED WITH $\alpha=0$ FOR THE TRACTION FREE, AXI-SYMMETRIC PENNY-SHAPED CRACK}

The series expansion (shadows) in (90) for the first three terms associated with $\alpha_{1}=\alpha_{2}=\alpha_{3}=0$ is explicitly derived herein.

For $\alpha_{1}=0$ :

$$
\phi_{0,1,0}=\left(\begin{array}{c}
\cos \varphi \\
-\sin \varphi \\
0
\end{array}\right), \quad \phi_{0,1,1}=-\cos \varphi\left(\begin{array}{c}
\cos \varphi \\
-\sin \varphi \\
0
\end{array}\right), \quad \phi_{0,1,2}=\cos ^{2} \varphi\left(\begin{array}{c}
\cos \varphi \\
-\sin \varphi \\
0
\end{array}\right), \quad \ldots
$$

For $\alpha_{2}=0$ :

$$
\begin{gathered}
\phi_{0,2,0}=\left(\begin{array}{c}
\sin \varphi \\
\cos \varphi \\
0
\end{array}\right), \quad \phi_{0,2, i}=0, \quad i \geq 1 \\
{\left[m_{0}\right] \phi_{0,2,0}=0,} \\
{\left[t_{0}\right] \phi_{0,2,0}=0 .}
\end{gathered}
$$

Furthermore $\left(2 \cos \varphi\left[m_{0}\right]+\left[m_{01}\right]\right) \phi_{0,2,0}=0$ and $\left(\cos \varphi\left[t_{0}\right]+\left[t_{01}\right]\right) \phi_{0,2,0}=0$.

Therefore $\phi_{0,2,1}=0$ is an admissible solution. Finally, since $\left(\cos ^{2} \varphi\left[m_{0}\right]+\cos \varphi\left[m_{01}\right]+\left[m_{02}\right]\right) \phi_{0,2,0}=$ 0 , the next term $\phi_{0,2,2}$ can be chosen as zero. Hence we have a solution without any shadow similar to the case of the Laplace operator.

For $\alpha_{3}=0$ :

We have

$$
\phi_{0,3,0}=\left(\begin{array}{c}
0 \\
0 \\
1
\end{array}\right), \quad \phi_{0,3,1}=\cos \varphi\left(\begin{array}{l}
0 \\
0 \\
1
\end{array}\right), \quad \phi_{0,3, i}=0, \quad i \geq 2
$$

$$
\begin{aligned}
{\left[m_{0}\right] \phi_{0,3,0} } & =0 \\
{\left[t_{0}\right] \phi_{0,3,0} } & =0
\end{aligned}
$$

Next, notice the disturbing fact is that

$$
\left(2 \cos \varphi\left[m_{0}\right]+\left[m_{01}\right]\right) \phi_{0,3,0}=0 \quad \text { and } \quad\left(\cos \varphi\left[t_{0}\right]+\left[t_{01}\right]\right) \phi_{0,3,0}=0
$$

letting the possibility of choosing $\phi_{0,3,1}=0$. But, with such a choice, since

$$
\left(\cos ^{2} \varphi\left[m_{0}\right]+\cos \varphi\left[m_{01}\right]+\left[m_{02}\right]\right) \phi_{0,3,0} \neq 0
$$

we would be obliged to calculate a non-zero $\phi_{0,3,2}$. Most presumably resulting in an infinite series of shadows.

The special circumstance is that there exists a particular solution with one shadow only if, instead of choosing $\phi_{0,3,1}=0$, we take

We have

$$
\phi_{0,3,1}=\cos \varphi\left(\begin{array}{l}
0 \\
0 \\
1
\end{array}\right)
$$

$$
\begin{aligned}
{\left[m_{0}\right] \boldsymbol{\phi}_{0,3,1} } & =-\left(2 \cos \varphi\left[m_{0}\right]+\left[m_{01}\right]\right) \boldsymbol{\phi}_{0,3,0}=0 \\
{\left[t_{0}\right] \phi_{0,3,1} } & =-\left(\cos \varphi\left[t_{0}\right]+\left[t_{01}\right]\right) \phi_{0,3,0}=0,
\end{aligned}
$$


and the new fact is that

$$
-\left(2 \cos \varphi\left[m_{0}\right]+\left[m_{01}\right]\right) \phi_{0,3,1}-\left(\cos ^{2} \varphi\left[m_{0}\right]+\cos \varphi\left[m_{01}\right]+\left[m_{02}\right]\right) \phi_{0,3,0}=0
$$

allowing the possibility to choose $\phi_{0,3,2}=0$. Since

$$
-\left(\cos ^{2} \varphi\left[m_{0}\right]+\cos \varphi\left[m_{01}\right]+\left[m_{02}\right]\right) \phi_{0,3,1}=0
$$

we see that the series of shadows can be stopped there. In this way we find a global rigid body motion as specified in Remark 8.

\section{REFERENCES}

[1] Bach, M., Nazarov, S.A., Wendland, W.L.: Propagation of a penny shaped crack under the Irwin criterion. In: Analysis, numerics and applications of differential and integral equations (Stuttgart, 1996), Pitman Res. Notes Math. Ser., vol. 379, pp. 17-21. Longman, Harlow (1998)

[2] Costabel, M., Dauge, M., Duduchava, R.: Asymptotics without logarithmic terms for crack problems. Communication in PDEs 28(5-6), 869-926 (2003)

[3] Costabel, M., Dauge, M., Yosibash, Z.: A quasidual function method for extracting edge stress intensity functions. SIAM Jour. Math. Anal. 35(5), 1177-1202 (2004)

[4] Dauge, M.: Elliptic boundary value problems in corner domains - smoothness and asymptotics of solutions. Lecture notes in Mathematics 1341, Springer-Verlag, Heidelberg (1988)

[5] Kondratiev, V.A.: Boundary value problems for elliptic equations in domains with conical or angular points. Transact. Moscow Math. Soc. 16, 227-313 (1967)

[6] Leblond, J., Torlai, O.: The stress-field near the front of an arbitrarily shaped crack in a 3-dimensional elastic body. Jour. of Elasticity 29(2), 97-131 (1992)

[7] Leung, A., Su, R.: Eigenfunction expansion for penny-shaped and circumferential cracks. Int. Jour. Fracture 89, 205-222 (1998)

[8] Omer, N., Yosibash, Z., Costabel, M., Dauge, M.: Edge flux intensity functions in polyhedral domains and their extraction by a quasidual function method. Int. Jour. Fracture 129, 97-130 (2004)

[9] von Petersdorff, T., Stephan, E.P.: Decompositions in edge and corner singularities for the solution of the Dirichlet problem of the Laplacian in a polyhedron. Math. Nachr. 149, 71-103 (1990)

[10] von Petersdorff, T., Stephan, E.P.: Singularities of the solution of the Laplacian in domains with circular edges. Appl. Anal. 45(1-4), 281-294 (1992)

[11] Sokolnikoff, I.S.: Mathematical Theory of Elasticity. McGraw-Hill, New York (1956)

[12] Williams, M.L.: Stress singularities resulting from various boundary conditions in angular corners of plates in extension. Trans. ASME, Jour. Appl. Mech. 19, 526-528 (1952)

[13] Yosibash, Z., Omer, N., Costabel, M., Dauge, M.: Edge stress intensity functions in polyhedral domains and their extraction by a quasidual function method. Int. Jour. Fracture 136, 37 - 73 (2005)

\section{ADDRESSES}

Z.Y. \& S.S.: Pearlstone Center for Aeronautical Eng. Studies, Dept. of Mechanical Engineering, BenGurion University of THE Negev, BeER-Sheva, 84105, Israel, Tel.: +972-8-6477103, FAX: +972-8-6477101 E-mail address: zohary@bgu. ac.il

M.D. \& M.C.: IRMAR, Université de Rennes 1, Campus de Beaulieu, 35042 Rennes Cedex, France

E-mail address: monique. dauge@univ-rennesi.fr

E-mail address: martin. costabeleuniv-rennesl.fr 